\title{
Synthesis and Evaluation of Cyclic Acetals of Serine Hydroxyl- amine for Amide-Forming KAHA Ligations
}

\author{
Simon Baldaufa \\ Jeffrey W. Bode*a,b \\ a Laboratorium für Organische Chemie, Department of Chemistry \\ and Applied Biosciences, ETH Zürich, 8093 Zürich, Switzerland \\ bode@org.chem.ethz.ch \\ b Institute of Transformative Bio-Molecules (WPI-ITbM), Nagoya \\ University, Chikusa, Nagoya 464-8602, Japan \\ Published as part of the 50 Years SYNTHESIS - Golden Anniversary Issue
}
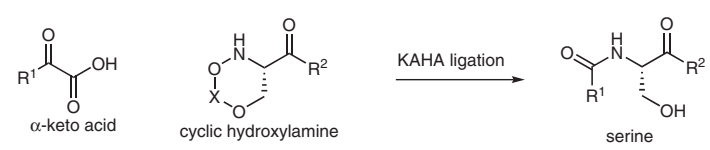

\author{
Received: 21.11.2018 \\ Accepted: 27.11.2018 \\ Published online: 07.01.2019 \\ DOI: 10.1055/s-0037-1611635; Art ID: ss-2018-z0777-op \\ License terms: CC $0 \$$
}

Abstract The $\alpha$-ketoacid-hydroxylamine (KAHA) ligation allows the coupling of unprotected peptide segments. The most widely used variant employs a 5-membered cyclic hydroxylamine that forms a homoserine ester as the primary ligation product. While very effective, monomers that give canonical amino acid residues are in high demand. In order to preserve the stability and reactivity of cyclic hydroxylamines, but form a canonical amino acid residue upon ligation, we sought to prepare cyclic derivatives of serine hydroxylamine. An evaluation of several cyclization strategies led to cyclobutanone ketals as the leading structures. The preparation, stability, and amide-forming ligation of these serine-derived ketals are described.

Key words ligation, hydroxylamines, acetals, amides, peptides

In 2006, we reported the $\alpha$-ketoacid-hydroxylamine (KAHA) amide-forming ligation, ${ }^{1}$ which complemented the well-established native chemical ligation ${ }^{2}(\mathrm{NCL})$ for the chemical synthesis of proteins. These methods allow chemoselective couplings of unprotected peptide segments for the synthesis of peptides and proteins. ${ }^{3}$ Currently the most used hydroxylamine for KAHA ligations is 5-oxaproline, due to its high stability and chemoselective reactivity with $\alpha-$ ketoacids to form a homoserine residue at the ligation site. ${ }^{4}$ The primary product of this reaction is an ester, which rearranges to an amide under basic conditions (Scheme 1$).^{5}$ In 2015, we developed a novel oxazetidine acid which gave serine at the ligation site, exclusively as the amide product. ${ }^{6}$ Unfortunately, the synthesis of this monomer is rather long and it is not stable in its unprotected form.

In this report, we describe the synthesis of a six-membered cyclic hydroxylamine, which directly yields a native



b) This work:<smiles>CCCC(=O)C(=O)OC</smiles>



Scheme 1 KAHA ligation with 5-oxaproline and proposed cyclic serine hydroxylamine

serine residue at the ligation site (Scheme 1). Furthermore, we describe our efforts to incorporate these novel monomers into a peptide segment.

In designing an alternative, serine-forming ligation monomer we sought to identify a larger ring structure that we hoped would be more easily prepared. This consideration led us to consider cyclic variants of L-serine hydroxylamine, including cyclic carbonates, cyclic sulfonates, and cyclic acetals. To begin, L-serine was converted into the corresponding ethyl ester 1 . TBS protection of the free alcohol and subsequent treatment with bromoacetonitrile in the presence of a base gave 2 .

This was treated with a slightly modified protocol from Fukuyama to give the $N$-hydroxyl intermediate 3 . $^{7}$ Initially we envisioned selective Boc-protection of the nitrogen, but this was unsuccessful under various conditions. ${ }^{8}$ We instead elected to first protect the free hydroxyl group using $\mathrm{TBSCl}$ followed by $\mathrm{N}$-protection with Fmoc-Cl to give 4. Although initial attempts to remove the TBS protecting group 
using TBAF led mainly to decomposition, treatment of $\mathbf{4}$ with concentrated $\mathrm{HCl}$ gave clean conversion into diol $\mathbf{5}$ (Scheme 2).

$$
\text { 1) } \mathrm{m}_{\mathrm{CPBA}, \mathrm{CH}_{2} \mathrm{Cl}_{2}}
$$

Scheme 2 Synthesis of core structure 5

We explored the formation of the corresponding cyclic boronic esters, silanes, carbonates, sulfites, and phosphoric acid esters; however, we were not able to isolate the corresponding six-membered cyclic hydroxylamines. As an alternative, we considered cyclic acetals, as similar structures have proven to be well suited for KAHA ligation. ${ }^{9}$ For use in protein synthesis, however, these acid-labile functional groups would need to survive cleavage from the resin under standard TFA conditions. Prior to in-depth investigations of suitable acetals, we carried out preliminary tests to check that cyclic acetals of $\mathbf{5}$ could be formed and that Fmoc-removal was feasible. We treated compound 5 with 1,1-dimethoxyethylbenzene in the presence of $p$-toluenesulfonic acid to give $\mathbf{6}$ without problem. Removal of the Fmoc group under basic conditions also proceeded smoothly. With this promising result we sought to prepare and evaluate various cyclic acetals.

We initially targeted a methylene acetal, as these are known to be more resistant to acid cleavage than other structures. ${ }^{10}$ Unfortunately, all attempts to form the desired compound from $\mathbf{5}$ with various methylene sources using different catalysts or activation using Brønsted or Lewis acids did not yield the desired product. Despite this setback, we continued to synthesize various acetals and ketals by using either a catalytic amount of $p$-toluenesulfonic acid for aldehyde-derived compounds, or $\operatorname{In}(\mathrm{OTf})_{3}$ for the ketone derivatives. Selected acetals and ketals prepared are shown in Table 1 . However, when these compounds were tested for their stability under SPPS conditions [resin cleavage conditions, TFA/DODT/ $\mathrm{H}_{2} \mathrm{O}(95: 2.5: 2.5 \mathrm{v} / \mathrm{v})$ for $2 \mathrm{~h}$ ], only cyclobutanone-derivative 12 showed reasonable stability (Table 1 ).

Despite the poor acid stability, we tested these substrates for activity in KAHA ligation. For this purpose, the Fmoc protecting group was removed with piperidine and the monomers were allowed to react with simple $\alpha$-ketoac- id 13 to give amide 14 (Table 2). Commonly reported KAHA conditions use DMSO/ $\mathrm{H}_{2} \mathrm{O}$ at $60{ }^{\circ} \mathrm{C}$, but a $\mathrm{HFIP} / \mathrm{AcOH}$ mixture showed much better solubility and the reaction was observed to proceed at $45{ }^{\circ} \mathrm{C} .{ }^{11}$ Therefore all ligation studies were carried out under these conditions. Almost all monomers tested showed good activity in the KAHA ligation. After a period of 12 hours, $\alpha$-ketoacid 13 was consumed. The benzylic acetals were stable under the acidic ligation conditions, and electron-poor nitrobenzyl acetal 16 gave slightly better conversion compared to bromobenzyl acetal 15.

Table 1 Synthesized Monomers and Their Stability ${ }^{a}$

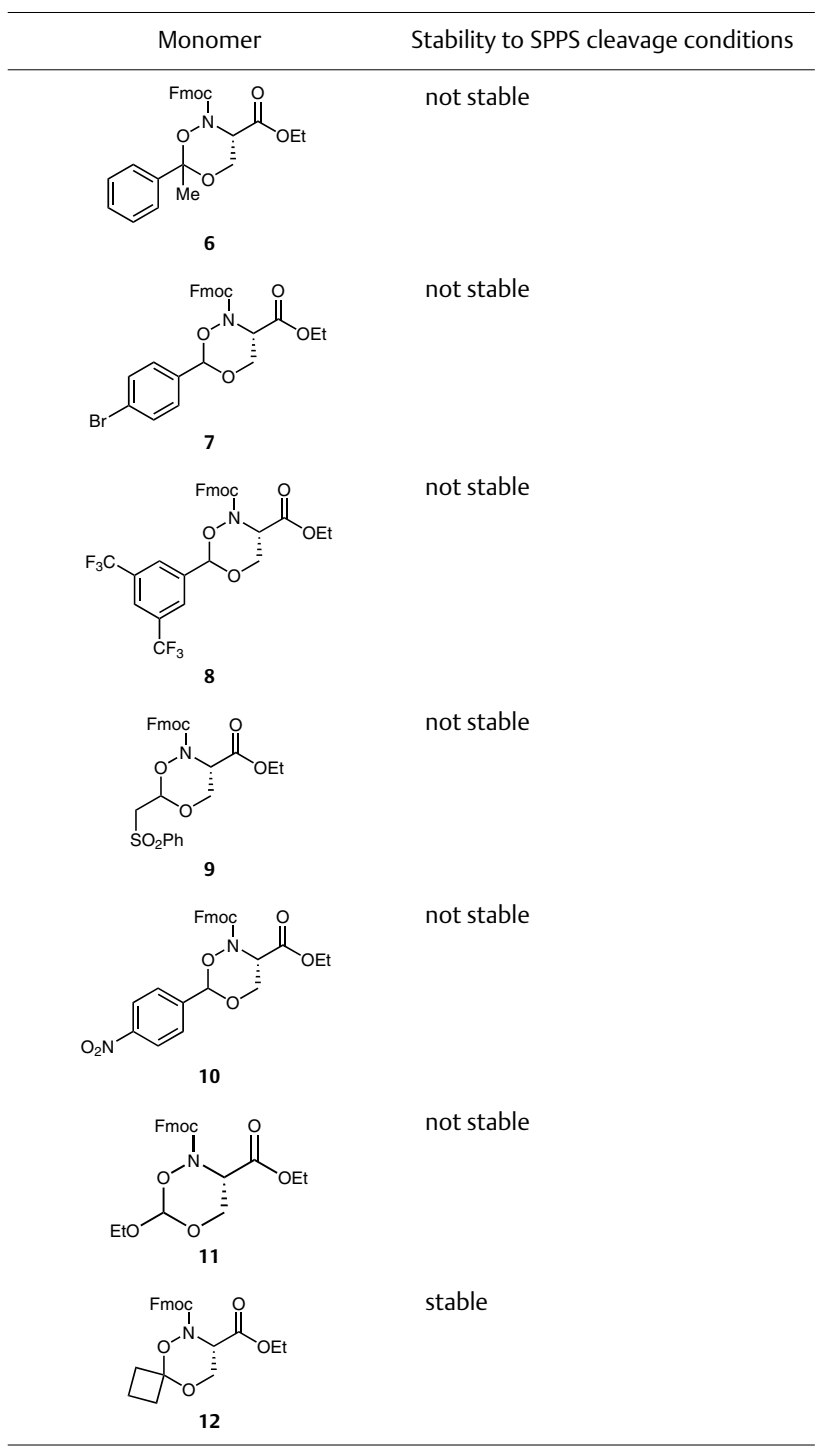

a Stability was tested by treatment with TFA/DODT/ $\mathrm{H}_{2} \mathrm{O}(95: 2.5: 2.5 \mathrm{v} / \mathrm{v})$ for $2 \mathrm{~h}$. 
Table 2 KAHA Ligation with Selected Monomers
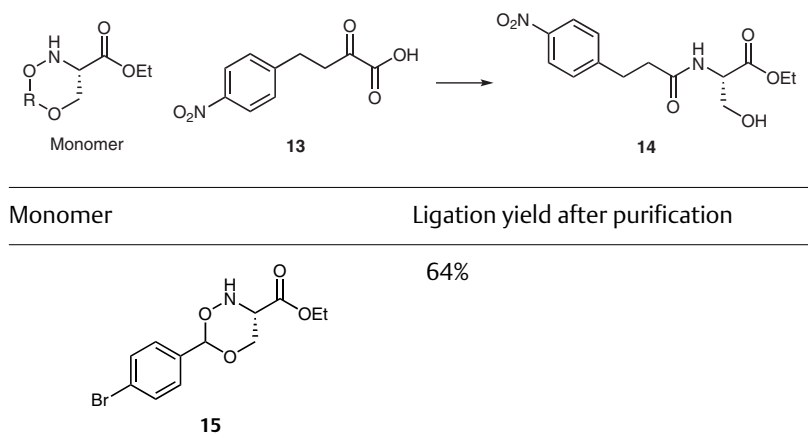<smiles>CC(=O)C1CNC(c2ccc([N+](=O)[O-])cc2)OC1</smiles>

$77 \%$<smiles>CCCC1CC(=O)NO1</smiles>

no product observed

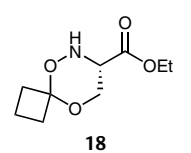

$72 \%$

monomer 18

monomer $16^{\mathrm{b}}$
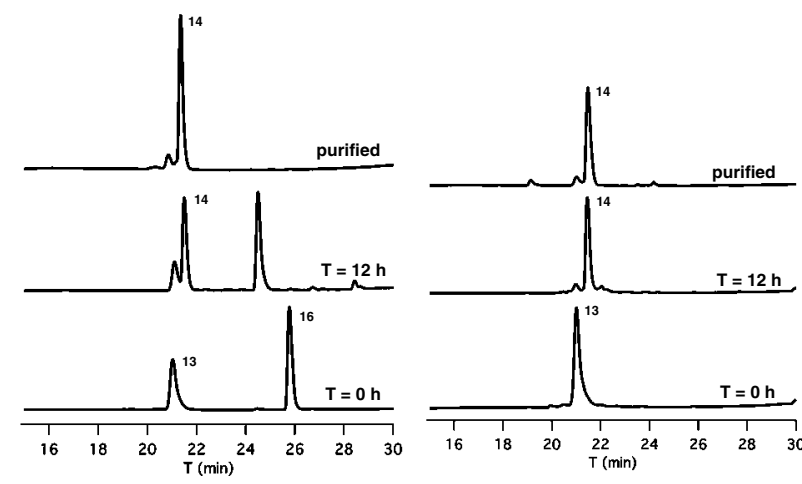

a $\mathrm{KAHA}$ ligation carried out at $30 \mathrm{mM}$ at $45^{\circ} \mathrm{C}$ in $\mathrm{HFIP} / \mathrm{AcOH}$ with $1 \% \mathrm{H}_{2} \mathrm{O}$ for $12 \mathrm{~h}$.

${ }^{\mathrm{b}} \mathrm{HPLC}$ monitoring of the KAHA ligation of $\alpha$-ketoacid 13 and hydroxylamine 16, and purified amide 14.

c HPLC monitoring of the KAHA ligation of $\alpha$-ketoacid 13 and hydroxylamine 18, and purified amide 14.

Cyclobutanone-derived acetal 18 was also stable during the ligation conditions and gave good conversion of the $\alpha$ ketoacid. Only 17 did not perform KAHA ligation under $\mathrm{HFIP} / \mathrm{AcOH}$ conditions because the deprotected monomer was not stable under the ligation conditions (Table 2).

This experiment also indicated that open, unsubstituted serine hydroxylamine does not react under these conditions and that the cyclic structure is essential. With these promising results, we sought to remove the ethyl ester in the presence of Fmoc and the acetal, for the purpose of applying this monomer in Fmoc-SPPS. Despite all our efforts, the various conditions only led to total decomposition or returned starting material without removing the Fmoc protecting group.

We instead introduced a benzyl ester at the beginning of the synthesis. All previously developed steps were compatible with the benzyl-protected starting material, and we were able to synthesize the benzyl ester analogue 19. Unfortunately, deprotection of the benzyl ester using Pd/C under a $\mathrm{H}_{2}$ atmosphere gave mainly decomposition and only traces of product was observed. We were pleased to see that exchanging $\mathrm{Pd} / \mathrm{C}$ for $\mathrm{Pd}(\mathrm{OH})_{2}$ allowed a clean reaction.

Free acid $\mathbf{2 6}$ was coupled onto a short peptide segment, but proved not to be stable upon resin cleavage. Therefore we prepared and evaluated a number of other ketals from small rings. Although we were not successful in forming the product from oxetan-3-one, other cyclobutanone derivatives proved tractable. Brominated derivative $\mathbf{2 0}$ was readily formed and carboxylic esters $\mathbf{2 1}$ and $\mathbf{2 2}$ could be prepared from the corresponding acid followed by ester formation. The stability of compounds 19-22 was tested on the monomer and on a peptide segment (Table 3 ).

Table 3 Synthesized Monomers and Their Stability ${ }^{a}$

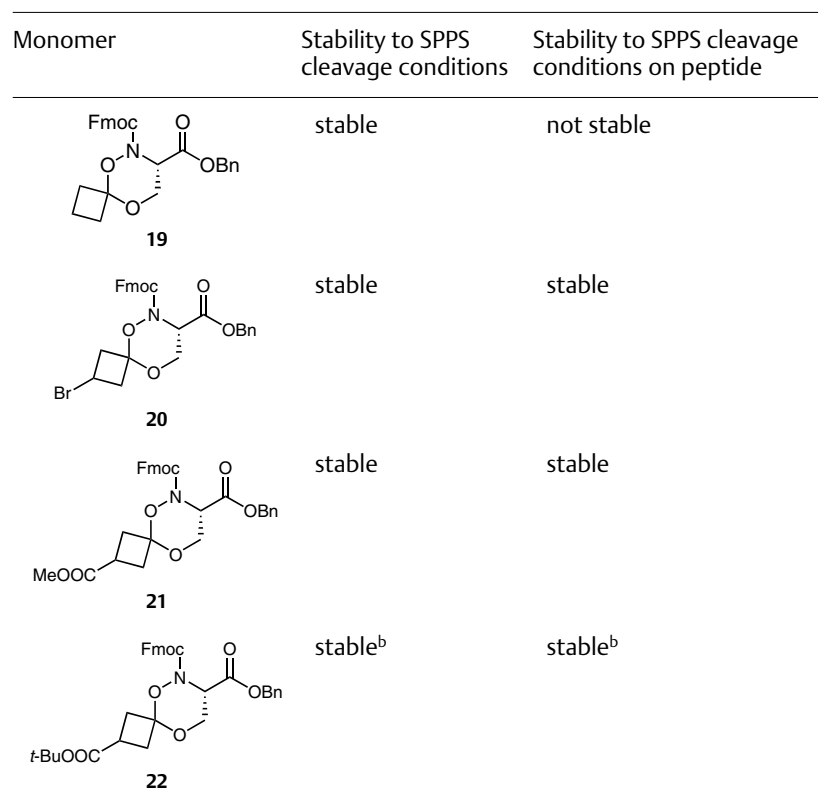

a Stability was tested by treatment with TFA/DODT/ $\mathrm{H}_{2} \mathrm{O}(95: 2.5: 2.5 \mathrm{v} / \mathrm{v})$ for $2 \mathrm{~h}$.

${ }^{b}$ Observed as free acid on the cyclobutane ring.

All benzyl-protected monomers were also tested for their performance in the KAHA ligation. Fmoc removal occurred smoothly in all cases and the deprotected monomers underwent ligation with $\alpha$-ketoacid 13 in $\mathrm{HFIP} / \mathrm{AcOH}$ (Table 4). The cyclobutanone ketal monomers could be converted into the corresponding acid by treatment with 
$\mathrm{Pd}(\mathrm{OH})_{2}$ under $\mathrm{H}_{2}$ atmosphere (Scheme 3) and all compounds tolerated the coupling conditions to introduce the monomer onto the $\mathrm{N}$-terminus of a peptide segment. Since OMe ester 21 showed better conversion in the KAHA ligation, we selected this monomer for introduction onto a short peptide sequence.

Table 4 KAHA Ligation with Selected Monomers ${ }^{\mathrm{a}}$
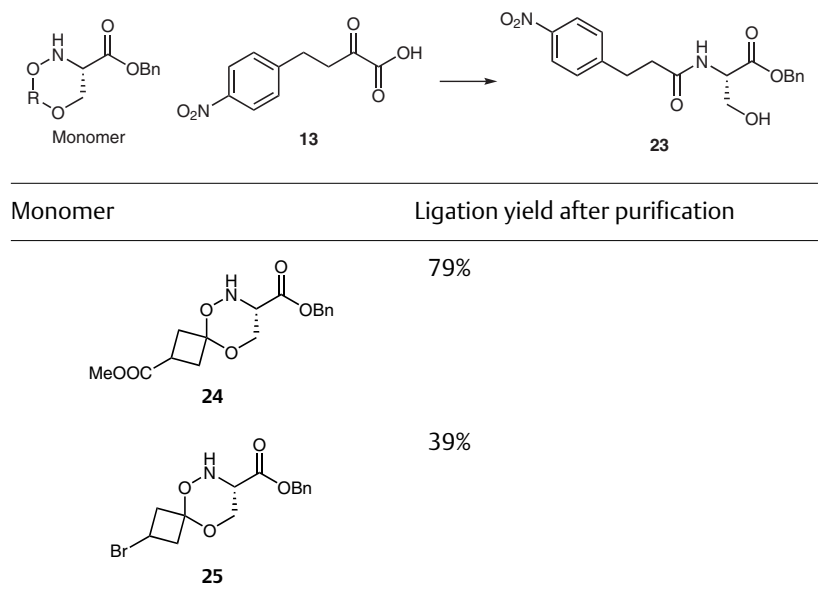

13 and $24^{\mathrm{b}}$

13 and $25^{c}$
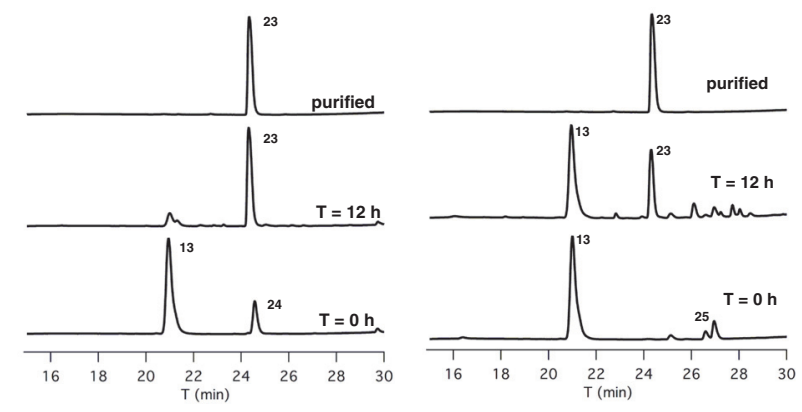

${ }^{a} \mathrm{KAHA}$ ligation carried out at $30 \mathrm{mM}$ at $45^{\circ} \mathrm{C}$ in $\mathrm{HFIP} / \mathrm{AcOH}$ with $1 \% \mathrm{H}_{2} \mathrm{O}$ for $12 \mathrm{~h}$.

${ }^{b}$ HPLC monitoring of the KAHA ligation of $\alpha$-ketoacid 13 and hydroxylamine 24, and purified amide 23.

' HPLC monitoring of the KAHA ligation of $\alpha$-ketoacid 13 and hydroxylamine 25, and purified amide 23.

A short peptide segment with three amino acid residues, prepared by standard SPPS conditions on a Rink amide resin, was chosen as a model peptide. After deprotection of the terminal amine, the free acid of monomer 27 was coupled with HATU/NMM conditions to the resin and cleaved with TFA/DODT/ $\mathrm{H}_{2} \mathrm{O}(95: 2.5: 2.5 \mathrm{v} / \mathrm{v})$ for 2 hours. TFA was removed at $40{ }^{\circ} \mathrm{C}$ under reduced pressure and the product was isolated by preparative HPLC. The Fmoc protecting group was removed with $\mathrm{N}, \mathrm{N}$-diethylamine and the

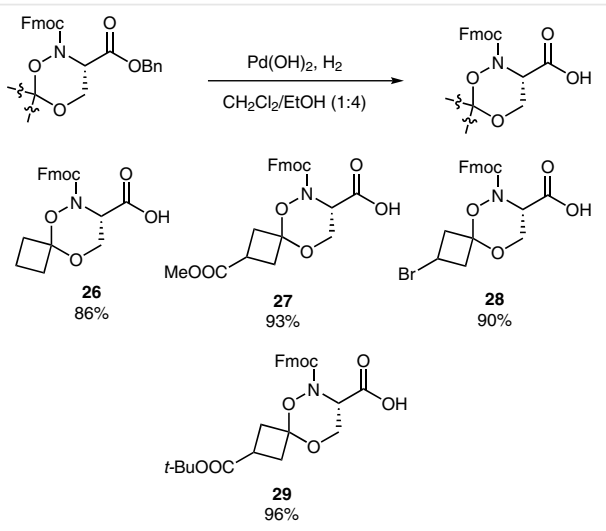

Scheme 3 Benzyl deprotection

KAHA ligation performed directly on the unprotected product without further purification using $\alpha$-ketoacid 13 in $\mathrm{HFIP} / \mathrm{AcOH}$. The free hydroxylamine $\mathbf{3 1}$ was observed by LCMS and was completely consumed within 12 hours (Scheme 4).
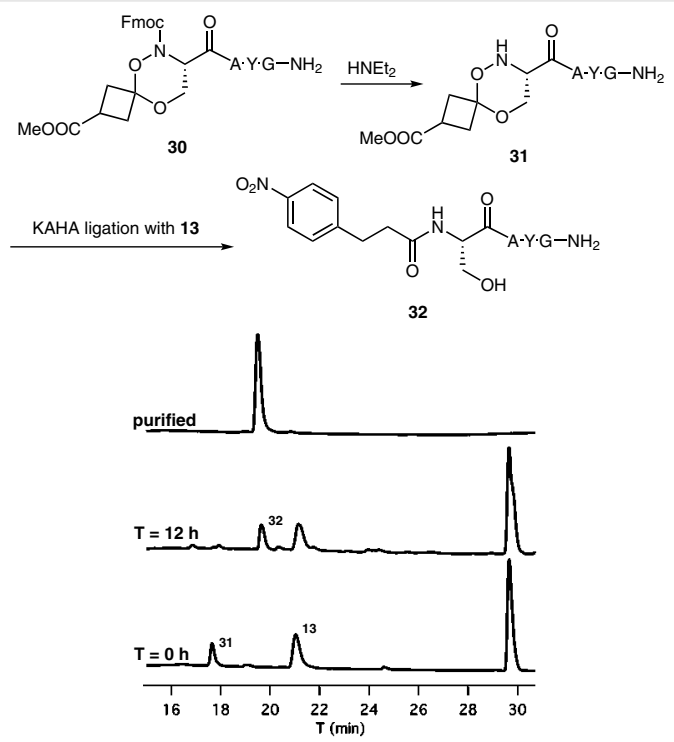

Scheme 4 Ligation test on a small peptide segment

After this positive result, we expanded to a larger peptide fragment consisting of 50 amino acid residues. Although the Fmoc group could be removed cleanly, with a peak-to-peak conversion by HPLC, the acetal protecting group unfortunately did not survive the two-step procedure of basic Fmoc-removal conditions and acidic purification. The obtained product was $\mathbf{3 6}$, the free hydroxylamine of serine at the N-terminus (Scheme 5). 




Scheme 5 Attempts to remove the Fmoc protecting group on a longer peptide fragment

The results were the same regardless of the choice of base used to remove the Fmoc protecting group, including basic aqueous solutions. To date, we were not able to overcome this problem and longer peptides containing the cyclic ketal serine hydroxylamines cannot be isolated. A search for a more stable acetal protecting group is currently under investigation.

In conclusion, we devised a synthetic route to new hydroxylamine monomers that yield serine residues upon KAHA ligation. We found that substituted cyclobutane ketals are stable under acidic conditions and are excellent ligation partners. Further investigations are necessary to apply this monomer on larger peptides, however this strategy provides a promising new approach to KAHA ligation that forms canonical amino acid residues at the ligation site.

Fmoc-amino acids with suitable side-chain protecting groups, HATU (1-[bis(dimethylamino)methylene]-1H-1,2,3-triazolo[4,5-b]pyridinium 3-oxide hexafluorophosphate) were purchased from Peptides International (Louisville, KY, USA) and ChemImpex (Wood Dale, IL, USA). Solvents for flash chromatography (EtOAc, hexanes, $\mathrm{MeOH}$ ) were of technical grade and distilled prior to use. HPLC grade MeCN from Sigma-Aldrich was used for analytical and preparative HPLC purification. DMF (>99.8\%) from Sigma-Aldrich was directly used without further purification for solid phase peptide synthesis. Other commercially available reagents and solvents were purchased from Sigma-Aldrich (Buchs, Switzerland), Acros Organics (Geel, Belgium), and TCI Europe (Zwijndrecht, Belgium). DODT $=2,2^{\prime}$-(ethylenedioxy)diethanethiol. ${ }^{1} \mathrm{H}$ and ${ }^{13} \mathrm{C}$ NMR spectra were recorded on Bruker DRX400, Bruker AVIII400 and Bruker AVIII600 spectrometers. HRMS were recorded by the Mass Service of the Laboratory of Organic Chemistry at ETH Zurich either with a Bruker maXis instrument (ESIMS measurements) equipped with an ESI source and a Qq-TOF detector or with a Bruker solariX instrument (MALDI-FTICR-MS) using 4hydroxy- $\alpha$-cyanocinnamic acid as matrix. All reactions were performed using standard techniques under an atmosphere of $\mathrm{N}_{2}$. Reactions and fractions from flash chromatography were monitored by TLC using aluminum plates (Merck, TLC Silica gel $60 \mathrm{~W} \mathrm{~F}_{254} \mathrm{~S}$ ) and visualized by staining with basic $\mathrm{KMnO}_{4}$ solution or acidic ninhydrin solution. Flash chromatography was performed on Silicycle $\mathrm{SiO}_{2}$ Type F60 (230-400 mesh) using a forced flow of air at 0.5-1.0 bar. Unless otherwise stated, peptides and protein segments were analyzed and purified by RP-HPLC on Jasco analytical and preparative instruments equipped with dual pumps, mixer and in-line degasser, a variable wavelength UV detector (simultaneous monitoring of the eluent at $220 \mathrm{~nm}, 254 \mathrm{~nm}$, and $301 \mathrm{~nm}$ ) and a Rheodyne injector fitted with a 20 or $1000 \mu \mathrm{L}$ injection loop. If required, the columns were heated using a column heater or a water bath (preparative HPLC). The mobile phase for RP-HPLC was Milipore- $\mathrm{H}_{2} \mathrm{O}$ containing $0.1 \%$ (v/v) TFA and HPLC grade MeCN containing 0.1\% (v/v) TFA. Analytical HPLC was performed on Shiseido Capcell Pak C18 MGII (5 $\mu \mathrm{m}$, $4.6 \mathrm{~mm}$ i.d. $\times 250$ $\mathrm{mm}$ ) or Shiseido Capcell Pak C18 (UG 80, $5 \mu \mathrm{m}, 4.6 \mathrm{~mm}$ i.d. $\times 250 \mathrm{~mm}$ ) columns at a flow rate of $1 \mathrm{~mL} / \mathrm{min}$. Preparative HPLC was performed on Shiseido Capcell Pak MGII ( $5 \mu \mathrm{m}, 20 \mathrm{~mm}$ i.d. $\times 250 \mathrm{~mm}$ ) or Vydac 248MS C18 $(10 \mu \mathrm{m}, 22 \mathrm{~mm}$ i.d. $\times 250 \mathrm{~mm})$ columns at a flow rate of $10 \mathrm{~mL} / \mathrm{min}$.

Ethyl O-(tert-Butyldimethylsilyl)- $\mathrm{N}$-(cyanomethyl)-L-serinate (2) IR (neat): 2954, 2930, 2857, 1733, 1471, 1251, $1195 \mathrm{~cm}^{-1}$.

${ }^{1} \mathrm{H}$ NMR (400 MHz, $\left.\mathrm{CDCl}_{3}\right): \delta=4.19(\mathrm{q}, J=7.1 \mathrm{~Hz}, 2 \mathrm{H}), 3.92-3.84(\mathrm{~m}, 2$ $\mathrm{H}), 3.80-3.59(\mathrm{~m}, 2 \mathrm{H}), 3.48(\mathrm{dt}, J=8.2,4.4 \mathrm{~Hz}, 1 \mathrm{H}), 2.28(\mathrm{q}, J=7.0 \mathrm{~Hz}$, $1 \mathrm{H}), 1.27$ (t, J = 7.2 Hz, $3 \mathrm{H}), 0.85(\mathrm{~s}, 9 \mathrm{H}), 0.03(\mathrm{~d}, J=5.7 \mathrm{~Hz}, 6 \mathrm{H})$.

$\left.{ }^{13} \mathrm{C} \mathrm{NMR} \mathrm{(101} \mathrm{MHz,} \mathrm{CDCl}_{3}\right): \delta=171.54(\mathrm{CO}), 117.73(\mathrm{CN}), 64.41\left(\mathrm{CH}_{2}\right)$, $61.61(\mathrm{CH}), 61.39\left(\mathrm{CH}_{2}\right), 36.07\left(\mathrm{CH}_{2}\right), 25.82\left(3 \mathrm{CH}_{3}\right), 18.27(\mathrm{C}), 14.29$ $\left(\mathrm{CH}_{3}\right),-5.41\left(\mathrm{CH}_{3}\right),-5.55\left(\mathrm{CH}_{3}\right)$.

HRMS (ESI): $m / z$ [M $+\mathrm{H}]^{+}$calcd for $\mathrm{C}_{13} \mathrm{H}_{27} \mathrm{~N}_{2} \mathrm{O}_{3} \mathrm{Si}$ : 287.1785; found: 287.1786 .

\section{Ethyl O-(tert-Butyldimethylsilyl)- $\mathrm{N}$-hydroxy-L-serinate (3)}

IR (neat): 2953, 2929, 2857, 1737, 1471, 1252, $1107 \mathrm{~cm}^{-1}$.

${ }^{1} \mathrm{H}$ NMR (400 MHz, $\mathrm{CDCl}_{3}$ ): $\delta=6.59$ (br s, $1 \mathrm{H}$ ), 5.83 (br s, $1 \mathrm{H}$ ), 4.21 (qd, $J=7.2,0.8 \mathrm{~Hz}, 2 \mathrm{H}$ ), 3.88 (qd, $J=10.2,4.8 \mathrm{~Hz}, 2 \mathrm{H}$ ), 3.74 (dd, $J=5.6$, $4.0 \mathrm{~Hz}, 1 \mathrm{H}), 1.27(\mathrm{t}, J=7.1 \mathrm{~Hz}, 3 \mathrm{H}), 0.85(\mathrm{~s}, 9 \mathrm{H}), 0.03(\mathrm{~d}, J=3.3 \mathrm{~Hz}, 6$ $\mathrm{H})$.

${ }^{13} \mathrm{C}$ NMR $\left(101 \mathrm{MHz}, \mathrm{CDCl}_{3}\right): \delta=171.21(\mathrm{CO}), 66.83(\mathrm{CH}), 61.21\left(\mathrm{CH}_{2}\right)$, $61.08\left(\mathrm{CH}_{2}\right), 25.85\left(3 \mathrm{CH}_{3}\right), 18.29(\mathrm{C}), 14.31\left(\mathrm{CH}_{3}\right),-5.46\left(\mathrm{CH}_{3}\right),-5.53$ $\left(\mathrm{CH}_{3}\right)$.

HRMS (ESI): $m / z$ [M $+\mathrm{H}]^{+}$calcd for $\mathrm{C}_{11} \mathrm{H}_{26} \mathrm{NO}_{4} \mathrm{Si}$ : 264.1626; found: 264.1627.

Ethyl $\mathrm{N}$-(tert-Butyldimethylsiloxy)-O-(tert-butyldimethylsilyl)- $\mathrm{N}$ [(9H-fluoren-9-ylmethoxy)carbonyl]-L-serinate (4)

IR (neat): 2953, 2928, 2856, 1738, 1463, 1249, $1095 \mathrm{~cm}^{-1}$.

${ }^{1} \mathrm{H}$ NMR $\left(400 \mathrm{MHz}, \mathrm{CDCl}_{3}\right): \delta=7.79(\mathrm{dt}, J=7.6,0.9 \mathrm{~Hz}, 2 \mathrm{H}), 7.69(\mathrm{dt}$, $J=7.5,1.0 \mathrm{~Hz}, 2 \mathrm{H}), 7.42(\mathrm{tt}, J=7.5,0.8 \mathrm{~Hz}, 2 \mathrm{H}), 7.32(\mathrm{tt}, J=7.4,1.1 \mathrm{~Hz}$, $2 \mathrm{H}), 4.72(\mathrm{dd}, J=7.9,6.3 \mathrm{~Hz}, 1 \mathrm{H}), 4.55-4.39(\mathrm{~m}, 2 \mathrm{H}), 4.31(\mathrm{t}, J=7.1$ $\mathrm{Hz}, 1 \mathrm{H}), 4.21(\mathrm{qd}, J=7.1,3.2 \mathrm{~Hz}, 2 \mathrm{H}), 4.10(\mathrm{dd}, J=7.2,1.8 \mathrm{~Hz}, 2 \mathrm{H})$, $1.28(\mathrm{t}, J=7.1 \mathrm{~Hz}, 3 \mathrm{H}), 0.96$ (s, $9 \mathrm{H}), 0.91(\mathrm{~s}, 9 \mathrm{H}), 0.21$ (d, $J=22.0 \mathrm{~Hz}, 6$ $\mathrm{H}), 0.09$ (s, $6 \mathrm{H})$.

${ }^{13} \mathrm{C}$ NMR (101 MHz, $\mathrm{CDCl}_{3}$ ): $\delta=168.68$ (CO), $159.38(\mathrm{CO}), 143.89(\mathrm{C})$, $143.82(\mathrm{C}), 141.44(\mathrm{C}), 141.42(\mathrm{C}), 127.87(\mathrm{CH}), 127.86(\mathrm{CH}), 127.21(2$ $\mathrm{CH}), 125.49(\mathrm{CH}), 125.43(\mathrm{CH}), 120.10(\mathrm{CH}), 120.09(\mathrm{CH}), 68.36\left(\mathrm{CH}_{2}\right)$, $66.93(\mathrm{CH}), 61.45\left(\mathrm{CH}_{2}\right), 59.85\left(\mathrm{CH}_{2}\right), 47.13(\mathrm{CH}), 26.09\left(3 \mathrm{CH}_{3}\right), 26.00$ $\left(3 \mathrm{CH}_{3}\right), 18.41(2 \mathrm{C}), 14.32\left(\mathrm{CH}_{3}\right),-4.70\left(\mathrm{CH}_{3}\right),-4.73\left(\mathrm{CH}_{3}\right),-5.21\left(\mathrm{CH}_{3}\right)$, $-5.29\left(\mathrm{CH}_{3}\right)$.

HRMS (ESI): $m / z[\mathrm{M}+\mathrm{H}]^{+}$calcd for $\mathrm{C}_{32} \mathrm{H}_{50} \mathrm{NO}_{6} \mathrm{Si}_{2}: 600.3171$; found: 600.3174 .

Ethyl $N$-[(9H-Fluoren-9-ylmethoxy)carbonyl]- $N$-hydroxy-L-serinate (5)

IR (neat): 2956, 1730, 1700, 1450, 1312, 1111, $1047 \mathrm{~cm}^{-1}$. 
${ }^{1} \mathrm{H}$ NMR $\left(400 \mathrm{MHz}, \mathrm{CDCl}_{3}\right): \delta=7.78(\mathrm{dt}, J=7.6,1.0 \mathrm{~Hz}, 2 \mathrm{H}), 7.62(\mathrm{t}, J=$ $6.2 \mathrm{~Hz}, 2 \mathrm{H}$ ), 7.42 (tt, J = 7.5, $1.3 \mathrm{~Hz}, 2 \mathrm{H}), 7.36-7.29$ (m, $2 \mathrm{H}), 4.86$ (s, 1 $\mathrm{H}), 4.57-4.42(\mathrm{~m}, 2 \mathrm{H}), 4.35-4.21(\mathrm{~m}, 3 \mathrm{H}), 4.21-4.06(\mathrm{~m}, 3 \mathrm{H}), 2.74(\mathrm{~s}$, $1 \mathrm{H}), 1.29$ (td, $J=7.1,5.9 \mathrm{~Hz}, 3 \mathrm{H})$.

${ }^{13} \mathrm{C}$ NMR (101 MHz, $\left.\mathrm{CDCl}_{3}\right): \delta=158.33(\mathrm{CO}), 143.54(2 \mathrm{C}) * 143.48(2$ C), $141.43(2 \mathrm{C})^{*}, 141.43(2 \mathrm{C}), 128.02(2 \mathrm{CH})^{*}, 128.02(2 \mathrm{CH}), 127.32$ $(2 \mathrm{CH})^{*}, 127.30(2 \mathrm{CH}), 125.25(2 \mathrm{CH})^{*}, 125.20(2 \mathrm{CH}), 120.19(2 \mathrm{CH})$, $69.06\left(\mathrm{CH}_{2}\right), 64.07(\mathrm{CH}), 62.29\left(\mathrm{CH}_{2}\right), 60.57\left(\mathrm{CH}_{2}\right), 47.02(\mathrm{CH}), 14.35$ $\left(\mathrm{CH}_{3}\right)^{*}, 14.26\left(\mathrm{CH}_{3}\right)$; ${ }^{*}$ signals of rotamers.

HRMS (MALDI): $m / z[\mathrm{M}+\mathrm{Na}]^{+}$calcd for $\mathrm{C}_{20} \mathrm{H}_{21} \mathrm{NO}_{6} \mathrm{Na}$ : 394.1261; found: 394.1261 .

\section{Benzyl O-(tert-Butyldimethylsilyl)- $N$-(cyanomethyl)-L-serinate (benzyl-2)}

IR (neat): 3674, 2987, 2956, 1736, 1463, 1406, 1252, $1083 \mathrm{~cm}^{-1}$.

${ }^{1} \mathrm{H} \mathrm{NMR}\left(400 \mathrm{MHz}, \mathrm{CDCl}_{3}\right): \delta=7.40-7.31(\mathrm{~m}, 5 \mathrm{H}), 5.19(\mathrm{~s}, 2 \mathrm{H}), 3.97-$ 3.87 (m, $2 \mathrm{H}), 3.81-3.71(\mathrm{~m}, 1 \mathrm{H}), 3.71-3.53(\mathrm{~m}, 2 \mathrm{H}), 2.31$ (br s, $1 \mathrm{H})$, $0.86(\mathrm{~s}, 9 \mathrm{H}), 0.02(\mathrm{~d}, J=10.2 \mathrm{~Hz}, 6 \mathrm{H})$.

${ }^{13} \mathrm{C} \mathrm{NMR}\left(101 \mathrm{MHz}, \mathrm{CDCl}_{3}\right): \delta=171.48(\mathrm{CO}), 135.39(\mathrm{C}), 128.76(2 \mathrm{CH})$, $128.60(\mathrm{CH}), 128.49(2 \mathrm{CH}), 117.71(\mathrm{CN}), 67.17\left(\mathrm{CH}_{2}\right), 64.37\left(\mathrm{CH}_{2}\right)$, $61.68(\mathrm{CH}), 36.07\left(\mathrm{CH}_{2}\right), 25.85\left(3 \mathrm{CH}_{3}\right), 18.29(\mathrm{C}),-5.40\left(\mathrm{CH}_{3}\right),-5.51$ $\left(\mathrm{CH}_{3}\right)$.

HRMS (ESI): $m / z[\mathrm{M}+\mathrm{Na}]^{+}$calcd for $\mathrm{C}_{18} \mathrm{H}_{28} \mathrm{~N}_{2} \mathrm{NaO}_{3} \mathrm{Si}$ : 371.1761 found 371.1761.

\section{Benzyl O-(tert-Butyldimethylsilyl)- $N$-hydroxy-L-serinate (benzyl- 3)}

IR (neat): 3674, 2987, 2900, 1739, 1462, 1252, 1192, 1105, $1066 \mathrm{~cm}^{-1}$. ${ }^{1} \mathrm{H} \mathrm{NMR}\left(400 \mathrm{MHz}, \mathrm{CDCl}_{3}\right): \delta=7.40-7.29(\mathrm{~m}, 5 \mathrm{H}), 5.87$ (br s, $1 \mathrm{H}$ ), 5.58 (br s, $1 \mathrm{H}), 5.21$ (s, $2 \mathrm{H}), 3.97-3.87(\mathrm{~m}, 2 \mathrm{H}), 3.81$ (dd, $J=5.2,4.1$ $\mathrm{Hz}, 1 \mathrm{H}), 0.86$ (s, $9 \mathrm{H}), 0.03$ (s, $3 \mathrm{H}), 0.02(\mathrm{~s}, 3 \mathrm{H})$.

${ }^{13} \mathrm{C} \mathrm{NMR}\left(101 \mathrm{MHz}, \mathrm{CDCl}_{3}\right): \delta=171.19(\mathrm{CO}), 135.58(\mathrm{C}), 128.74(2 \mathrm{CH})$, $128.52(\mathrm{CH}), 128.41(2 \mathrm{CH}), 66.97\left(\mathrm{CH}_{2}\right), 66.90(\mathrm{CH}), 61.18\left(\mathrm{CH}_{2}\right)$, $25.89\left(3 \mathrm{CH}_{3}\right), 18.33(\mathrm{C}),-5.43\left(\mathrm{CH}_{3}\right),-5.48\left(\mathrm{CH}_{3}\right)$.

HRMS (ESI): $m / z[\mathrm{M}+\mathrm{H}]^{+}$calcd for $\mathrm{C}_{16} \mathrm{H}_{28} \mathrm{NO}_{4} \mathrm{Si}$ : 326.1782; found: 326.1779 .

\section{Benzyl $N$-(tert-Butyldimethylsiloxy)-O-(tert-butyldimethylsilyl)- $\mathbf{N}$-[(9H-fluoren-9-ylmethoxy)carbonyl]-L-serinate (benzyl-4)}

IR (neat): 3674, 2956, 1740, 1450, 1361, 1250, 1098, 1076, $1005 \mathrm{~cm}^{-1}$. ${ }^{1} \mathrm{H} \mathrm{NMR}\left(400 \mathrm{MHz}, \mathrm{CDCl}_{3}\right): \delta=7.75$ (dq, $J=7.5,0.9 \mathrm{~Hz}, 2 \mathrm{H}$ ), 7.63-7.59 (m, $2 \mathrm{H}), 7.46-7.18(\mathrm{~m}, 9 \mathrm{H}), 5.23-5.10(\mathrm{~m}, 2 \mathrm{H}), 4.71(\mathrm{dd}, J=7.6,6.5$ $\mathrm{Hz}, 1 \mathrm{H}), 4.49-4.35(\mathrm{~m}, 2 \mathrm{H}), 4.19(\mathrm{t}, J=7.0 \mathrm{~Hz}, 1 \mathrm{H}), 4.14-4.07(\mathrm{~m}, 2$ H), 0.93 (s, $9 \mathrm{H}), 0.88$ (s, $9 \mathrm{H}), 0.20(\mathrm{~s}, 3 \mathrm{H}), 0.15(\mathrm{~s}, 3 \mathrm{H}), 0.07(\mathrm{~s}, 3 \mathrm{H})$, $0.06(\mathrm{~s}, 3 \mathrm{H})$.

${ }^{13} \mathrm{C}$ NMR $\left(101 \mathrm{MHz}, \mathrm{CDCl}_{3}\right): \delta=168.55$ (CO), 159.37 (CO), 143.85 (2 C)*, $143.78(2 \mathrm{C}), 141.41(2 \mathrm{C})^{*}, 141.38(2 \mathrm{C}), 135.47(\mathrm{C}), 128.62(2$ $\mathrm{CH}), 128.36(\mathrm{CH}), 128.27(2 \mathrm{CH}), 127.82(2 \mathrm{CH})^{*}, 127.80(2 \mathrm{CH}), 127.19$ $(2 \mathrm{CH}), 125.40(2 \mathrm{CH})^{*}, 125.35(2 \mathrm{CH}), 120.06(2 \mathrm{CH})^{*}, 120.04(2 \mathrm{CH})$, $68.29\left(\mathrm{CH}_{2}\right), 67.16(\mathrm{CH}), 67.08\left(\mathrm{CH}_{2}\right), 59.96\left(\mathrm{CH}_{2}\right), 47.08(\mathrm{CH}), 26.08(3$ $\left.\mathrm{CH}_{3}\right), 25.99\left(3 \mathrm{CH}_{3}\right), 18.39(\mathrm{C}), 18.37(\mathrm{C}),-4.74\left(2 \mathrm{CH}_{3}\right),-5.23\left(\mathrm{CH}_{3}\right)$, $-5.30\left(\mathrm{CH}_{3}\right) ;{ }^{*}$ signals of rotamers.

HRMS (ESI): $m / z[M+H]^{+}$calcd for $\mathrm{C}_{37} \mathrm{H}_{52} \mathrm{NO}_{6} \mathrm{Si}_{2}: 662.3328$; found: 662.3323.
Benzyl $\mathrm{N}$-[(9H-Fluoren-9-ylmethoxy)carbonyl]- $\mathrm{N}$-hydroxy-L-serinate (benzyl-5)

IR (neat): 3674, 3305, 2969, 2900, 1738, 1708, 1450, 1262, 1106, 1048 $\mathrm{cm}^{-1}$.

${ }^{1} \mathrm{H}$ NMR $\left(400 \mathrm{MHz}, \mathrm{CDCl}_{3}\right): \delta=7.73(\mathrm{ddt}, J=7.6,2.1,0.9 \mathrm{~Hz}, 2 \mathrm{H}), 7.53$ $(\mathrm{t}, J=8.3 \mathrm{~Hz}, 2 \mathrm{H}), 7.37$ (tdt, $J=7.5,3.3,0.8 \mathrm{~Hz}, 2 \mathrm{H}), 7.35-7.17(\mathrm{~m}, 7$ H), 5.18 (s, 2 H), 4.88 (br s, 1 H), 4.47-4.34 (m, 2 H), 4.22-4.07 (m, 3 H), 3.13 (br s, $1 \mathrm{H}$ ); $1 \mathrm{H}$ not observed.

${ }^{13} \mathrm{C} \mathrm{NMR}\left(101 \mathrm{MHz}, \mathrm{CDCl}_{3}\right): \delta=168.65(\mathrm{CO}), 158.95(\mathrm{CO}), 143.39(2 \mathrm{C})$, 141.37 (2 C), $134.97(\mathrm{C}), 128.76(2 \mathrm{CH}), 128.65(\mathrm{CH}), 128.22(2 \mathrm{CH})$, $127.99(2 \mathrm{CH})^{*}, 127.97(2 \mathrm{CH}), 127.31(2 \mathrm{CH})^{*}, 127.29(2 \mathrm{CH}), 125.21$ $(2 \mathrm{CH})^{*}, 125.16(2 \mathrm{CH}), 120.14(2 \mathrm{CH}), 69.16\left(\mathrm{CH}_{2}\right), 67.70\left(\mathrm{CH}_{2}\right), 64.31$ $(\mathrm{CH}), 59.96\left(\mathrm{CH}_{2}\right), 46.91(\mathrm{CH})$; * signals of rotamers.

HRMS (ESI): $m / z$ [M $+\mathrm{Na}]^{+}$calcd for $\mathrm{C}_{25} \mathrm{H}_{23} \mathrm{NNaO}_{6}: 456.1418$; found: 456.1419 .

\section{Ketal Formation with In(OTf) $)_{3}$; General Procedure}

$\operatorname{In}(\mathrm{OTf})_{3}(0.30$ equiv) was added to a solution of $\mathbf{5}$ ( 1.00 equiv) and ketone (5.00 equiv) in $\mathrm{CH}_{2} \mathrm{Cl}_{2}(0.15 \mathrm{M})$ at $0{ }^{\circ} \mathrm{C}$. The reaction was allowed to warm up to r.t. and was stirred overnight. The solution was diluted with $\mathrm{CH}_{2} \mathrm{Cl}_{2}$ and $10 \%$ citric acid solution was added. The phases were separated and the aqueous layer was extracted with $\mathrm{CH}_{2} \mathrm{Cl}_{2}(2 \times)$. The combined organic layers were washed with sat. aq $\mathrm{NaHCO}_{3}$ solution and brine and dried $\left(\mathrm{Na}_{2} \mathrm{SO}_{4}\right)$. The drying agent was removed by filtration and the solvent was evaporated. The residue was purified by flash chromatography.

3-Ethyl 2-(9H-Fluoren-9-ylmethyl) (3S)-6-Methyl-6-phenyl-1,5,2dioxazinane-2,3-dicarboxylate (6)

IR (neat): 2987, 2900, 1713, 1449, 1304, 1098, $1065 \mathrm{~cm}^{-1}$.

${ }^{1} \mathrm{H} \mathrm{NMR}\left(500 \mathrm{MHz}, \mathrm{CDCl}_{3}\right): \delta=7.78(\mathrm{ddt}, J=7.6,5.7,0.9 \mathrm{~Hz}, 2 \mathrm{H}), 7.77-$ 7.62 (m, 2 H), 7.56-7.52 (m, 2 H), 7.46-7.27 (m, 7 H), 4.76-4.65 (m, 2 $\mathrm{H}), 4.45(\mathrm{dd}, J=10.5,7.7 \mathrm{~Hz}, 1 \mathrm{H}), 4.38(\mathrm{dd}, J=3.9,1.6 \mathrm{~Hz}, 2 \mathrm{H}), 4.33(\mathrm{t}$, $J=7.1 \mathrm{~Hz}, 1 \mathrm{H}$ ), 4.20 (qd, $J=7.1,1.6 \mathrm{~Hz}, 2 \mathrm{H}), 1.78(\mathrm{~s}, 3 \mathrm{H}), 1.20(\mathrm{t}, J=$ $7.1 \mathrm{~Hz}, 3 \mathrm{H})$.

${ }^{13} \mathrm{C}$ NMR (126 MHz, $\mathrm{CDCl}_{3}$ ): $\delta=168.07$ (CO), $156.52(\mathrm{CO}), 144.05(\mathrm{C})$, $143.43(\mathrm{C}), 141.57$ (C), $141.46(\mathrm{C}), 140.67(\mathrm{C}), 128.82(\mathrm{CH}), 128.30(2$ $\mathrm{CH}), 127.97(2 \mathrm{CH})^{*}, 127.92(2 \mathrm{CH}), 127.25(2 \mathrm{CH})^{*}, 127.20(2 \mathrm{CH})$, $125.66(\mathrm{CH}), 125.35(2 \mathrm{CH}), 125.24(\mathrm{CH}), 120.14(2 \mathrm{CH})^{*}, 120.10(2$ $\mathrm{CH}), 103.89(\mathrm{C}), 68.23\left(\mathrm{CH}_{2}\right), 62.16\left(\mathrm{CH}_{2}\right), 59.91\left(\mathrm{CH}_{2}\right), 57.53(\mathrm{CH})$, $47.14(\mathrm{CH}), 21.58\left(\mathrm{CH}_{3}\right), 14.22\left(\mathrm{CH}_{3}\right)$; ${ }^{*}$ signals of rotamers/diastereomers.

HRMS (MALDI): $m / z$ [M $+\mathrm{H}]^{+}$calcd for $\mathrm{C}_{28} \mathrm{H}_{28} \mathrm{NO}_{6}$ : 474.1911; found: 474.1907.

\section{7-Ethyl 6-(9H-Fluoren-9-ylmethyl) (S)-5,9-Dioxa-6- azaspiro[3.5]nonane-6,7-dicarboxylate (12)}

IR (neat): 3674, 2987, 2901, 1714, 1450, 1408, 1313, $1058 \mathrm{~cm}^{-1}$.

${ }^{1} \mathrm{H}$ NMR $\left(500 \mathrm{MHz}, \mathrm{CDCl}_{3}\right): \delta=7.83-7.78(\mathrm{~m}, 2 \mathrm{H}), 7.78-7.62(\mathrm{~m}, 2 \mathrm{H})$, 7.48-7.41 (m, 2 H), 7.38-7.31 (m, 2 H), 4.66 (dd, $J=10.3,6.6 \mathrm{~Hz}, 1 \mathrm{H})$, $4.55(\mathrm{~s}, 1 \mathrm{H}), 4.43(\mathrm{t}, J=9.2 \mathrm{~Hz}, 1 \mathrm{H}), 4.40-4.33(\mathrm{~m}, 2 \mathrm{H}), 4.30(\mathrm{q}, J=7.1$ $\mathrm{Hz}, 2 \mathrm{H}$ ), 4.05 (dd, $J=11.5,3.8 \mathrm{~Hz}, 1 \mathrm{H}), 2.49-2.36$ (m, $2 \mathrm{H}$ ), 2.36-2.24 (m, $2 \mathrm{H}), 1.99-1.80(\mathrm{~m}, 2 \mathrm{H}), 1.29(\mathrm{t}, J=7.1 \mathrm{~Hz}, 3 \mathrm{H})$.

${ }^{13} \mathrm{C} \mathrm{NMR}\left(126 \mathrm{MHz}, \mathrm{CDCl}_{3}\right): \delta=168.06$ (CO), $156.43(\mathrm{CO}), 144.05(2$ C)*, $143.37(2 \mathrm{C}), 141.50(2 \mathrm{C})^{*}, 141.43(2 \mathrm{C}), 127.98(2 \mathrm{CH})^{*}, 127.93(2$ $\mathrm{CH}), 127.25(2 \mathrm{CH})^{*}, 127.12(2 \mathrm{CH}), 125.58(2 \mathrm{CH})^{*}, 125.18(2 \mathrm{CH})$, $120.15(2 \mathrm{CH})^{*}, 120.13(2 \mathrm{CH}), 104.99(\mathrm{C}), 68.50\left(\mathrm{CH}_{2}\right), 62.34\left(\mathrm{CH}_{2}\right)$, $60.27\left(\mathrm{CH}_{2}\right), 57.22(\mathrm{CH}), 47.03(\mathrm{CH}), 32.70\left(\mathrm{CH}_{2}\right), 30.37\left(\mathrm{CH}_{2}\right), 14.23$ $\left(\mathrm{CH}_{3}\right), 11.59\left(\mathrm{CH}_{2}\right)$; * signals of rotamers/diastereomers. 
HRMS (ESI): $m / z[\mathrm{M}+\mathrm{Na}]^{+}$calcd for $\mathrm{C}_{24} \mathrm{H}_{25} \mathrm{NNaO}_{6}$ : 446.1574; found: 446.1575.

\section{7-Benzyl 6-(9H-Fluoren-9-ylmethyl) (S)-5,9-Dioxa-6-} azaspiro[3.5]nonane-6,7-dicarboxylate (19)

IR (neat): 3674, 2987, 2900, 1746, 1716, 1451, 1407, 1260, 1098, 1065 $\mathrm{cm}^{-1}$.

${ }^{1} \mathrm{H}$ NMR $\left(500 \mathrm{MHz}, \mathrm{CDCl}_{3}\right): \delta=7.77(\mathrm{dq}, J=7.6,1.1 \mathrm{~Hz}, 2 \mathrm{H}), 7.75-7.54$ (m, $2 \mathrm{H}), 7.47-7.21(\mathrm{~m}, 9 \mathrm{H}), 5.25(\mathrm{~s}, 2 \mathrm{H}), 4.56(\mathrm{dd}, J=10.4,6.7 \mathrm{~Hz}, 2$ $\mathrm{H}), 4.40-4.31(\mathrm{~m}, 2 \mathrm{H}), 4.22(\mathrm{t}, J=7.4 \mathrm{~Hz}, 1 \mathrm{H}), 4.17-4.00(\mathrm{~m}, 1 \mathrm{H})$, 2.50-2.19 (m, $4 \mathrm{H}), 1.99-1.78(\mathrm{~m}, 2 \mathrm{H})$.

${ }^{13} \mathrm{C} \mathrm{NMR}\left(126 \mathrm{MHz}, \mathrm{CDCl}_{3}\right.$ ): $\delta=168.05$ (CO), 156.45 (CO), $144.47(2$ C)*, $144.05(2 \mathrm{C})^{*}, 143.34(2 \mathrm{C}), 141.66(2 \mathrm{C})^{*}, 141.49\left(2 \mathrm{C}^{*}\right), 141.41(2$ C), $135.22(\mathrm{C}), 128.63(2 \mathrm{CH}), 128.44(\mathrm{CH}), 128.09(2 \mathrm{CH}), 127.97(2$ $\mathrm{CH})^{*}, 127.91(2 \mathrm{CH})^{*}, 127.73(2 \mathrm{CH}), 127.24(2 \mathrm{CH})^{*}, 127.11(2 \mathrm{CH})$, $125.60(2 \mathrm{CH})^{*}, 125.18(2 \mathrm{CH}), 124.84(2 \mathrm{CH}), 120.20(2 \mathrm{CH})^{*}, 120.13$ $(2 \mathrm{CH})^{*}, 120.10(2 \mathrm{CH}), 105.02(\mathrm{C}), 68.47\left(\mathrm{CH}_{2}\right), 67.87\left(\mathrm{CH}_{2}\right), 60.30$ $\left(\mathrm{CH}_{2}\right), 57.34(\mathrm{CH}), 46.96(\mathrm{CH}), 32.72\left(\mathrm{CH}_{2}\right), 30.41\left(\mathrm{CH}_{2}\right), 11.60\left(\mathrm{CH}_{2}\right)$; * signals of rotamers/diastereomers.

HRMS (ESI): $m / z\left[\mathrm{M}+\mathrm{NH}_{4}\right]^{+}$calcd for $\mathrm{C}_{29} \mathrm{H}_{31} \mathrm{~N}_{2} \mathrm{O}_{6}$ : 503.2177; found: 503.2174.

\section{7-Benzyl 6-(9H-Fluoren-9-ylmethyl) (S)-2-Bromo-5,9-dioxa-6- azaspiro[3.5]nonane-6,7-dicarboxylate (20)}

IR (neat): 3674, 2987, 2900, 1732, 1720, 1406, 1393, 1275, 1065, 1056 $\mathrm{cm}^{-1}$.

${ }^{1} \mathrm{H}$ NMR $\left(400 \mathrm{MHz}, \mathrm{CDCl}_{3}\right.$ ): $\delta=7.76$ (ddd, $J=8.4,2.7,1.0 \mathrm{~Hz}, 2 \mathrm{H}$ ), 7.55 (dd, $J=33.2,7.5 \mathrm{~Hz}, 2 \mathrm{H}), 7.43-7.22(\mathrm{~m}, 9 \mathrm{H}), 5.19(\mathrm{~s}, 2 \mathrm{H}), 4.62-4.32$ (m, $4 \mathrm{H}), 4.24-4.04(\mathrm{~m}, 2 \mathrm{H}), 3.90(\mathrm{dd}, J=11.6,3.7 \mathrm{~Hz}, 1 \mathrm{H}), 3.03-2.87$ (m, $2 \mathrm{H}), 2.70-2.46(\mathrm{~m}, 2 \mathrm{H})$.

${ }^{13} \mathrm{C}$ NMR (101 MHz, $\mathrm{CDCl}_{3}$ ): $\delta=167.56(\mathrm{CO}), 156.01(\mathrm{CO}), 143.81(2$ C)*, 143.15 (2 C), $141.53(2 \mathrm{C})^{*}, 141.44$ (2 C), 135.04 (C), 128.65 (2 $\mathrm{CH}), 128.52(\mathrm{CH}), 128.12(2 \mathrm{CH}), 128.03(2 \mathrm{CH})^{*}, 128.01(2 \mathrm{CH}), 127.26$ $(2 \mathrm{CH})^{*}, 127.19(2 \mathrm{CH}), 125.19(2 \mathrm{CH})^{*}, 124.94(2 \mathrm{CH}), 120.20(2 \mathrm{CH})^{*}$, $120.12(2 \mathrm{CH}), 102.37(\mathrm{C}), 67.97\left(\mathrm{CH}_{2}\right), 65.98\left(\mathrm{CH}_{2}\right), 60.86\left(\mathrm{CH}_{2}\right), 57.26$ $(\mathrm{CH}), 47.07(\mathrm{CH}), 45.50\left(\mathrm{CH}_{2}\right), 43.97\left(\mathrm{CH}_{2}\right), 31.60(\mathrm{CH})$; * signals of rotamers/diastereomers.

HRMS (MALDI/ESI): $m / z[\mathrm{M}+\mathrm{Na}]^{+}$calcd for $\mathrm{C}_{29} \mathrm{H}_{26} \mathrm{BrNNaO}_{6}$ : 586.0836; found: 586.0835 .

(S)-7-(Benzyloxycarbonyl)-6-[(9H-fluoren-9-ylmethoxy)carbonyl]-5,9-dioxa-6-azaspiro[3.5]nonane-2-carboxylic Acid (21a) IR (neat): 3674, 2987, 2900, 1736, 1450, 1393, 1264, 1105, $1065 \mathrm{~cm}^{-1}$. ${ }^{1} \mathrm{H}$ NMR (400 MHz, $\mathrm{CDCl}_{3}$ ): $\delta=10.67$ (br s, $\left.1 \mathrm{H}\right), 7.81-7.73(\mathrm{~m}, 2 \mathrm{H}$ ), 7.72-7.52 (m, $2 \mathrm{H}), 7.48-7.21(\mathrm{~m}, 9 \mathrm{H}), 5.25$ (s, $2 \mathrm{H}), 4.72-4.15(\mathrm{~m}, 5$ H), 4.11-3.91 (m, $1 \mathrm{H}), 3.09-2.95(\mathrm{~m}, 1 \mathrm{H}), 2.84-2.38$ (m, $4 \mathrm{H})$.

${ }^{13} \mathrm{C}$ NMR (101 MHz, $\left.\mathrm{CDCl}_{3}\right): \delta=179.80(\mathrm{CO}) * 179.74(\mathrm{CO}), 167.74$ $(\mathrm{CO})^{*}, 167.67(\mathrm{CO}), 156.57(\mathrm{CO})^{*}, 156.20(\mathrm{CO}), 143.80(2 \mathrm{C})^{*}, 143.74(2$ C)*, $143.32(2 \mathrm{C})^{*}, 143.14(2 \mathrm{C}), 141.43(2 \mathrm{C})^{*}, 141.34(2 \mathrm{C})^{*}, 141.29(2$ C)*, $141.22(2 \mathrm{C}), 135.03(\mathrm{C})^{*}, 134.99(\mathrm{C}), 128.57(2 \mathrm{CH})^{*}, 128.55(2$ $\mathrm{CH}), 128.42(\mathrm{CH})^{*}, 128.40(\mathrm{CH}), 128.02(2 \mathrm{CH})^{*}, 128.01(2 \mathrm{CH}), 127.95$ $(2 \mathrm{CH})^{*}, 127.93(2 \mathrm{CH})^{*}, 127.87(2 \mathrm{CH})^{*}, 127.83(2 \mathrm{CH}), 127.24(2 \mathrm{CH})^{*}$, $127.19(2 \mathrm{CH})^{*}, 127.10(2 \mathrm{CH}), 125.52(2 \mathrm{CH})^{*}, 125.31(2 \mathrm{CH})^{*}, 125.26$ $(2 \mathrm{CH})^{*}, 124.95(2 \mathrm{CH}), 120.10(2 \mathrm{CH})^{*}, 120.08(2 \mathrm{CH})^{*}, 119.96(2 \mathrm{CH})$, 101.56 (C), $68.25\left(\mathrm{CH}_{2}\right)^{*}, 67.90\left(\mathrm{CH}_{2}\right)^{*}, 67.87\left(\mathrm{CH}_{2}\right), 60.53\left(\mathrm{CH}_{2}\right)^{*}, 60.42$ $\left(\mathrm{CH}_{2}\right), 57.44(\mathrm{CH}), 46.91(\mathrm{CH})^{*}, 46.72(\mathrm{CH}), 36.34\left(\mathrm{CH}_{2}\right)^{*}, 36.15\left(\mathrm{CH}_{2}\right)$, $34.65\left(\mathrm{CH}_{2}\right)^{*}, 34.21\left(\mathrm{CH}_{2}\right), 28.84(\mathrm{C}), 28.71(\mathrm{CH})$; * signals of rotamers/diastereomers.
HRMS (ESI): $m / z[\mathrm{M}+\mathrm{H}]^{+}$calcd for $\mathrm{C}_{30} \mathrm{H}_{28} \mathrm{NO}_{8}$ : 530.1809; found: 530.1808 .

7-Benzyl 6-(9H-Fluoren-9-ylmethyl) 2-Methyl (S)-5,9-Dioxa-6azaspiro[3.5]nonane-2,6,7-tricarboxylate (21)

IR (neat): 3674, 2987, 2900, 1729, 1450, 1406, 1285, 1090, $1064 \mathrm{~cm}^{-1}$. ${ }^{1} \mathrm{H}$ NMR $\left(400 \mathrm{MHz}, \mathrm{CDCl}_{3}\right): \delta=7.80-7.75(\mathrm{~m}, 2 \mathrm{H}), 7.62$ (ddd, $J=41.3$, 13.0, 8.3 Hz, $2 \mathrm{H}), 7.46-7.20(\mathrm{~m}, 9 \mathrm{H}), 5.24(\mathrm{~d}, J=3.1 \mathrm{~Hz}, 2 \mathrm{H}), 4.65-$ $4.34(\mathrm{~m}, 3 \mathrm{H}), 4.36-4.15(\mathrm{~m}, 2 \mathrm{H}), 4.09-3.94(\mathrm{~m}, 1 \mathrm{H}), 3.71$ (d, $J=23.0$ $\mathrm{Hz}, 3 \mathrm{H}), 3.11-2.94(\mathrm{~m}, 1 \mathrm{H}), 2.85-2.40(\mathrm{~m}, 4 \mathrm{H})$.

${ }^{13} \mathrm{C}$ NMR (101 MHz, $\left.\mathrm{CDCl}_{3}\right): \delta=174.67(\mathrm{CO})^{*}, 174.35(\mathrm{CO}), 171.26$ (CO), $167.85(\mathrm{CO})^{*}, 167.75$ (CO), $143.93(2 \mathrm{C})^{*}, 143.37(2 \mathrm{C})^{*}, 143.25(2$ C), $141.50(2 \mathrm{C})^{*}, 141.43(2)^{*}, 141.33(2 \mathrm{C}), 135.11(\mathrm{C}), 128.65(2 \mathrm{CH})^{*}$, $128.63(2 \mathrm{CH}), 128.50(\mathrm{CH})^{*}, 128.48(\mathrm{CH}), 128.12(2 \mathrm{CH}), 128.01(2$ $\mathrm{CH})^{*}, 127.97(2 \mathrm{CH})^{*}, 127.93(2 \mathrm{CH}), 127.36(2 \mathrm{CH})^{*}, 127.28(2 \mathrm{CH})^{*}$, $127.26(2 \mathrm{CH})^{*}, 127.15(2 \mathrm{CH}), 125.72(2 \mathrm{CH})^{*}, 125.37(2 \mathrm{CH})^{*}, 125.05$ $(2 \mathrm{CH}), 120.17(2 \mathrm{CH})^{*}, 120.14(2 \mathrm{CH})^{*}, 120.08(2 \mathrm{CH})^{*}, 120.05(2 \mathrm{CH})$, $101.79(\mathrm{C}), 101.57(\mathrm{C}), 68.90\left(\mathrm{CH}_{2}\right)^{*}, 68.36\left(\mathrm{CH}_{2}\right), 67.98\left(\mathrm{CH}_{2}\right)^{*}, 67.95$ $\left(\mathrm{CH}_{2}\right), 60.63\left(\mathrm{CH}_{2}\right)^{*}, 60.53\left(\mathrm{CH}_{2}\right), 57.57(\mathrm{CH})^{*}, 57.37(\mathrm{CH}), 52.26\left(\mathrm{CH}_{3}\right)$, $46.99(\mathrm{CH})^{*}, 46.84(\mathrm{CH}), 36.57\left(\mathrm{CH}_{2}\right)^{*}, 36.42\left(\mathrm{CH}_{2}\right), 34.73\left(\mathrm{CH}_{2}\right)^{*}, 34.28$ $\left(\mathrm{CH}_{2}\right), 28.85(\mathrm{C}), 28.67(\mathrm{CH})$; * signals of rotamers/diastereomers.

HRMS (MALDI/ESI): $m / z[\mathrm{M}+\mathrm{Na}]^{+}$calcd for $\mathrm{C}_{31} \mathrm{H}_{29} \mathrm{NNaO}_{8}: 566.1785$; found: 566.1786 .

7-Benzyl 2-tert-Butyl 6-(9H-Fluoren-9-ylmethyl) (S)-5,9-Dioxa-6azaspiro[3.5]nonane-2,6,7-tricarboxylate (22)

IR (neat): 3674, 2974, 2900, 1722, 1451, 1366, 1283, 1157, 1089, 1012 $\mathrm{cm}^{-1}$.

${ }^{1} \mathrm{H}$ NMR (400 MHz, MeOD): $\delta=7.79$ (ddt, $J=7.6,3.1,0.9 \mathrm{~Hz}, 2 \mathrm{H}$ ), 7.61 (dt, $J=27.2,7.6 \mathrm{~Hz}, 2 \mathrm{H}), 7.51-7.14(\mathrm{~m}, 9 \mathrm{H}), 5.39-5.02$ (m, $3 \mathrm{H}), 4.67-$ 3.89 (m, 5 H), 2.98-2.14 (m, 5 H), 1.51-1.40 (m, 9 H).

${ }^{13} \mathrm{C}$ NMR (101 MHz, MeOD): $\delta=175.06(\mathrm{CO})^{*}, 174.93(\mathrm{CO}), 169.28$ (CO)*, 169.17 (CO), 157.46 (CO), $145.13(2 \mathrm{C})^{*}, 144.99(2 \mathrm{C})^{*}, 144.70(2$ C), $142.69(2 \mathrm{C})^{*}, 142.63(2 \mathrm{C})^{*}, 142.56(2 \mathrm{C})^{*}, 142.51$ (2 C), 136.75 $(\mathrm{C})^{*}, 136.69(\mathrm{C}), 129.53(2 \mathrm{CH})^{*}, 129.49(2 \mathrm{CH})^{*}, 129.47(2 \mathrm{CH}), 129.28$ $(\mathrm{CH}), 129.08(2 \mathrm{CH})^{*}, 129.03(2 \mathrm{CH})^{*}, 128.96(2 \mathrm{CH}), 128.91(2 \mathrm{CH})^{*}$, $128.90(2 \mathrm{CH}), 128.31(2 \mathrm{CH})^{*}, 128.26(2 \mathrm{CH})^{*}, 128.22(2 \mathrm{CH})^{*}, 128.17$ $(2 \mathrm{CH}), 126.52(2 \mathrm{CH})^{*}, 126.29(2 \mathrm{CH})^{*}, 126.15(2 \mathrm{CH})^{*}, 125.96(2 \mathrm{CH})$, $120.98(2 \mathrm{CH})^{*}, 120.94(2 \mathrm{CH}), 102.82(\mathrm{C})^{*}, 102.75(\mathrm{C}), 81.99(\mathrm{C})^{*}$, $81.93(\mathrm{C}), 69.71(\mathrm{CH}), 69.02\left(\mathrm{CH}_{2}\right)^{*}, 68.62\left(\mathrm{CH}_{2}\right), 68.56\left(\mathrm{CH}_{2}\right)^{*}, 67.91$ $\left(\mathrm{CH}_{2}\right), 61.73\left(\mathrm{CH}_{2}\right)^{*}, 61.44\left(\mathrm{CH}_{2}\right), 59.36(\mathrm{CH})^{*}, 58.99(\mathrm{CH}), 48.22(\mathrm{CH})^{*}$, $47.98(\mathrm{CH}), 37.03\left(\mathrm{CH}_{2}\right)^{*}, 36.86\left(\mathrm{CH}_{2}\right), 35.34\left(\mathrm{CH}_{2}\right)^{*}, 34.85\left(\mathrm{CH}_{2}\right), 31.19$ $(\mathrm{CH})^{*}, 30.93(\mathrm{CH})^{*}, 30.59(\mathrm{CH}), 28.29\left(3 \mathrm{CH}_{3}\right)^{*}, 28.25\left(3 \mathrm{CH}_{3}\right)$; ${ }^{*}$ signals of rotamers/diastereomers.

HRMS (MALDI/ESI): $m / z[\mathrm{M}+\mathrm{Na}]^{+}$calcd for $\mathrm{C}_{34} \mathrm{H}_{35} \mathrm{NNaO}_{8}: 608.2255$; found: 608.2257 .

6-[(9H-Fluoren-9-yl)methyl] 2-Methyl (S)-7-\{(S)-3-[2-\{(S)-1-[(2Amino-2-oxoethyl)amino]-3-(4-hydroxyphenyl)-1-oxopropan-2yl\}hydrazineyl]-2-oxobutanoyl\}-5,9-dioxa-6-azaspiro[3.5]nonane2,6-dicarboxylate (30)

HRMS (ESI): $m / z$ [M + Na] $]^{+}$calcd for $\mathrm{C}_{38} \mathrm{H}_{41} \mathrm{~N}_{5} \mathrm{NaO}_{11}$ : 766.2695; found: 766.2696.

HPLC (Shiseido Capcell Pak UG80 C18 column $(4.6 \times 250 \mathrm{~mm})$, heated to $60{ }^{\circ} \mathrm{C}, 10$ to $90 \% \mathrm{CH}_{3} \mathrm{CN}$ with $0.1 \%$ TFA in $20 \mathrm{~min}$ ): $t_{\mathrm{R}}=24.8 \mathrm{~min}$.

\section{Segment 33}

HRMS (ESI): $m / z$ [M] ${ }^{+}$calcd for $\mathrm{C}_{282} \mathrm{H}_{431} \mathrm{BrN}_{70} \mathrm{O}_{83} \mathrm{~S}_{2}$ : 6269.0282; found: 6269.0453. 
HPLC (Shiseido Capcell Pak UG80 C18 column (4.6 $\times 250 \mathrm{~mm}$ ), heated to $60{ }^{\circ} \mathrm{C}, 10$ to $90 \% \mathrm{CH}_{3} \mathrm{CN}$ with $0.1 \% \mathrm{TFA}$ in $20 \mathrm{~min}$ ): $t_{\mathrm{R}}=24.9 \mathrm{~min}$.

\section{Segment 34}

HRMS (ESI): $m / z$ [M] $]^{+}$calcd for $\mathrm{C}_{284} \mathrm{H}_{434} \mathrm{~N}_{70} \mathrm{O}_{85} \mathrm{~S}_{2}$ : 6249.1231; found: 6249.0065

HPLC (Shiseido Capcell Pak UG80 C18 column (4.6 $\times 250 \mathrm{~mm}$ ), heated to $60{ }^{\circ} \mathrm{C}, 10$ to $90 \% \mathrm{CH}_{3} \mathrm{CN}$ with $0.1 \% \mathrm{TFA}$ in $20 \mathrm{~min}$ ): $t_{\mathrm{R}}=24.5 \mathrm{~min}$.

\section{Segment 35}

HRMS (ESI): $m / z$ [M] $]^{+}$calcd for $\mathrm{C}_{284} \mathrm{H}_{433} \mathrm{~N}_{69} \mathrm{O}_{85} \mathrm{~S}_{2}$ : 6234.1122; found: 6235.1309

HPLC (Shiseido Capcell Pak UG80 C18 column $(4.6 \times 250 \mathrm{~mm})$, heated to $60{ }^{\circ} \mathrm{C}, 10$ to $90 \% \mathrm{CH}_{3} \mathrm{CN}$ with $0.1 \%$ TFA in $20 \mathrm{~min}$ ): $t_{\mathrm{R}}=24.1 \mathrm{~min}$.

\section{Acetal Formation with p-Toluenesulfonic Acid Monohydrate; Gen- eral Procedure \\ Dimethyl acetal (2.00 equiv) and $p$-toluenesulfonic acid monohydrate ( 0.10 equiv) were added to 5 ( 1.00 equiv) in DMF $(0.05 \mathrm{M})$. The solu- tion was stirred at $50{ }^{\circ} \mathrm{C}$ under vacuum for $4 \mathrm{~h}$. The resulting dark brown viscous gel was diluted with $\mathrm{CH}_{2} \mathrm{Cl}_{2}$ and sat. aq $\mathrm{NaHCO}_{3}$ solu- tion was added to the solution. The aqueous layer was extracted with $\mathrm{CH}_{2} \mathrm{Cl}_{2}(2 \times)$. The combined organic layers were washed with brine and dried $\left(\mathrm{MgSO}_{4}\right)$. The drying agent was removed by filtration and the solvent was evaporated. The residue was purified by flash chro- matography.}

\section{3-Ethyl 2-(9H-Fluoren-9-ylmethyl) (3S)-6-(4-Bromophenyl)-1,5,2-} dioxazinane-2,3-dicarboxylate (7)

IR (neat): 3674, 2986, 2900, 1719, 1449, 1304, 1209, $1084 \mathrm{~cm}^{-1}$.

${ }^{1} \mathrm{H}$ NMR $\left(400 \mathrm{MHz}, \mathrm{CDCl}_{3}\right): \delta=7.79-7.20(\mathrm{~m}, 12 \mathrm{H}), 5.66(\mathrm{~s}, 1 \mathrm{H}), 4.80-$ $4.63(\mathrm{~m}, 3 \mathrm{H}), 4.49(\mathrm{t}, J=9.9 \mathrm{~Hz}, 1 \mathrm{H}), 4.34-4.23(\mathrm{~m}, 3 \mathrm{H}), 4.20-4.06$ (m, $1 \mathrm{H}), 1.26(\mathrm{t}, J=7.1 \mathrm{~Hz}, 3 \mathrm{H})$.

${ }^{13} \mathrm{C}$ NMR (101 MHz, CDCl $)$ ): $\delta=167.75(\mathrm{CO}), 156.42(\mathrm{CO}), 143.92(\mathrm{C})$, $143.11(\mathrm{C}), 141.54(\mathrm{C}), 141.42(\mathrm{C}), 133.85(\mathrm{C}), 131.67(2 \mathrm{CH}), 128.42(2$ $\mathrm{CH}), 128.02(2 \mathrm{CH})^{*}, 127.97(2 \mathrm{CH}), 127.22(2 \mathrm{CH}), 125.66(2 \mathrm{CH})^{*}$, $125.39(2 \mathrm{CH}), 124.21(\mathrm{CBr}), 120.16(2 \mathrm{CH}), 103.06(\mathrm{CH}), 68.35\left(\mathrm{CH}_{2}\right)$, $66.12\left(\mathrm{CH}_{2}\right), 62.41\left(\mathrm{CH}_{2}\right), 57.26(\mathrm{CH}), 47.12(\mathrm{CH}), 14.27\left(\mathrm{CH}_{3}\right) ;$; signals of rotamers/diastereomers.

HRMS (ESI): $m / z\left[\mathrm{M}+\mathrm{NH}_{4}\right]^{+}$calcd for $\mathrm{C}_{27} \mathrm{H}_{28} \mathrm{BrN}_{2} \mathrm{O}_{6}$ : 555.1125; found: 555.1118 .

3-Ethyl 2-(9H-Fluoren-9-ylmethyl) (3S)-6-[3,5-Bis(trifluoromethyl)phenyl]-1,5,2-dioxazinane-2,3-dicarboxylate (8)

IR (neat): 2970, 2901, 1727, 1450, 1277, 1175, $1132 \mathrm{~cm}^{-1}$.

${ }^{1} \mathrm{H}$ NMR (400 MHz, $\left.\mathrm{CDCl}_{3}\right): \delta=8.22-7.21(\mathrm{~m}, 9 \mathrm{H}), 5.82(\mathrm{br} \mathrm{s}, 1 \mathrm{H}$ ), 4.90-4.66 (m, $3 \mathrm{H}), 4.58$ (dd, $J=10.6,7.4 \mathrm{~Hz}, 1 \mathrm{H}), 4.38-4.24(\mathrm{~m}, 3 \mathrm{H})$, 4.22-4.09 (m, $1 \mathrm{H}), 1.33-1.20(\mathrm{~m}, 3 \mathrm{H}) ; 2 \mathrm{H}_{\text {arom }}$ not observed.

${ }^{13} \mathrm{C}$ NMR (101 MHz, $\mathrm{CDCl}_{3}$ ): $\delta=167.51(\mathrm{CO}), 141.54(\mathrm{C}), 132.02(\mathrm{q}, J=$ $\left.33.9 \mathrm{~Hz}, 2 \mathrm{CF}_{3}\right), 128.08(\mathrm{CH}), 127.27(\mathrm{CH}), 125.30(\mathrm{CH}), 123.83(\mathrm{CH})$, $120.15(\mathrm{CH}), 101.54(\mathrm{CH}), 68.71\left(\mathrm{CH}_{2}\right), 66.24\left(\mathrm{CH}_{2}\right), 62.62\left(\mathrm{CH}_{2}\right), 57.55$, $47.14(\mathrm{CH}), 14.24\left(\mathrm{CH}_{3}\right)$; several $\mathrm{C}$ not assigned due to mixture of diastereomers and rotamers.

$\left.{ }^{19} \mathrm{~F} \mathrm{NMR} \mathrm{(377} \mathrm{MHz,} \mathrm{CDCl}_{3}\right): \delta=-62.80$.

HRMS (ESI): $m / z$ [M $\left.+\mathrm{NH}_{4}\right]^{+}$calcd for $\mathrm{C}_{29} \mathrm{H}_{27} \mathrm{~F}_{6} \mathrm{~N}_{2} \mathrm{O}_{6}$ : 613.1768; found: 613.1769 .
3-Ethyl 2-(9H-Fluoren-9-ylmethyl) (3S)-6-[(Phenylsulfonyl)methyl]-1,5,2-dioxazinane-2,3-dicarboxylate (9)

IR (neat): 2987, 1736, 1447, 1306, 1147, 1116, $1065 \mathrm{~cm}^{-1}$.

${ }^{1} \mathrm{H}$ NMR $\left(500 \mathrm{MHz}, \mathrm{CDCl}_{3}\right): \delta=8.11-7.24(\mathrm{~m}, 13 \mathrm{H}), 5.05$ (ddd, $J=20.8$, 6.6, $4.1 \mathrm{~Hz}, 1 \mathrm{H}), 4.50$ (ddd, $J=14.1,10.4,7.3 \mathrm{~Hz}, 1 \mathrm{H}), 4.45-4.29(\mathrm{~m}, 2$ H), 4.28-4.19 (m, $2 \mathrm{H}), 4.17-4.00(\mathrm{~m}, 0.5 \mathrm{H}), 3.95$ (dd, $J=10.1,4.2 \mathrm{~Hz}$, $0.5 \mathrm{H}), 3.54(\mathrm{dt}, J=14.5,7.4 \mathrm{~Hz}, 1 \mathrm{H}), 3.39(\mathrm{dt}, J=14.3,4.2 \mathrm{~Hz}, 1 \mathrm{H})$, $3.31(\mathrm{~d}, J=6.1 \mathrm{~Hz}, 2 \mathrm{H}), 1.27(\mathrm{q}, J=7.1 \mathrm{~Hz}, 3 \mathrm{H})$.

${ }^{13} \mathrm{C}$ NMR (126 MHz, $\mathrm{CDCl}_{3}$ ): $\delta=168.26(\mathrm{CO}), 157.70(\mathrm{CO}), 143.93(\mathrm{C})$, 143.70 (C)*, 141.42 (C), 141.36 (C), 139.67 (C), 139.48 (C), 134.18 $(\mathrm{CH}), 129.47(2 \mathrm{CH}), 128.15(2 \mathrm{CH}), 128.03(\mathrm{CH}), 127.89(\mathrm{CH}), 127.26$ (2 $\mathrm{CH}), 125.62(\mathrm{CH}), 125.42(\mathrm{CH}), 120.08(2 \mathrm{CH}), 98.92(\mathrm{CH})^{*}, 97.72$ $(\mathrm{CH}), 68.79\left(\mathrm{CH}_{2}\right)^{*}, 68.72\left(\mathrm{CH}_{2}\right), 62.22\left(\mathrm{CH}_{2}\right)^{*}, 62.19\left(\mathrm{CH}_{2}\right)^{*}, 61.94$ $\left(\mathrm{CH}_{2}\right), 59.35\left(\mathrm{CH}_{2}\right)^{*}, 58.27\left(\mathrm{CH}_{2}\right), 53.83\left(\mathrm{CH}_{2}\right), 47.05(\mathrm{CH}), 14.27\left(\mathrm{CH}_{3}\right)$; * signals of rotamers/diastereomers.

HRMS (ESI): $m / z[\mathrm{M}+\mathrm{H}]^{+}$calcd for $\mathrm{C}_{28} \mathrm{H}_{28} \mathrm{NO}_{8} \mathrm{~S}$ : 538.1530 ; found: 538.1524 .

3-Ethyl 2-(9H-Fluoren-9-ylmethyl) (3S)-6-(4-Nitrophenyl)-1,5,2dioxazinane-2,3-dicarboxylate (10)

IR (neat): 3674, 2987, 2901, 1724, 1524, 1450, 1349, 1242, $1080 \mathrm{~cm}^{-1}$. ${ }^{1} \mathrm{H}$ NMR $\left(500 \mathrm{MHz}, \mathrm{CDCl}_{3}\right): \delta=8.26-8.21(\mathrm{~m}, 2 \mathrm{H}), 7.81-7.52(\mathrm{~m}, 6 \mathrm{H})$, 7.45-7.27 (m, $4 \mathrm{H}), 5.67(\mathrm{~s}, 1 \mathrm{H}), 4.74(\mathrm{~s}, 2 \mathrm{H}), 4.59$ (dd, $J=10.6,7.2 \mathrm{~Hz}$, $1 \mathrm{H}), 4.28$ (dq, $J=14.2,6.9 \mathrm{~Hz}, 3 \mathrm{H}), 4.19-4.04(\mathrm{~m}, 2 \mathrm{H}), 1.25$ (t, $J=7.1$ $\mathrm{Hz}, 3 \mathrm{H})$.

${ }^{13} \mathrm{C}$ NMR (126 MHz, $\mathrm{CDCl}_{3}$ ): $\delta=167.55$ (CO), 156.33 (CO), 148.85 $\left(\mathrm{CNO}_{2}\right), 143.90(2 \mathrm{C})^{*}, 143.09(2 \mathrm{C}), 141.59(2 \mathrm{C})^{*}, 141.44(\mathrm{C}), 141.00$ (2 C), $128.07(2 \mathrm{CH})^{*}, 128.04(2 \mathrm{CH}), 127.77(2 \mathrm{CH}), 127.24(2 \mathrm{CH})$, $125.42(2 \mathrm{CH})^{*}, 125.29(2 \mathrm{CH}), 123.63(2 \mathrm{CH}), 120.21(2 \mathrm{CH})^{*}, 120.17$ $(2 \mathrm{CH}), 101.98(\mathrm{CH}), 68.23\left(\mathrm{CH}_{2}\right), 66.17\left(\mathrm{CH}_{2}\right), 62.51\left(\mathrm{CH}_{2}\right), 57.34(\mathrm{CH})$, $47.19(\mathrm{CH}), 14.26\left(\mathrm{CH}_{3}\right)$; ${ }^{*}$ signals of rotamers/diastereomers.

HRMS (ESI): $m / z[\mathrm{M}+\mathrm{Na}]^{+}$calcd for $\mathrm{C}_{27} \mathrm{H}_{24} \mathrm{~N}_{2} \mathrm{NaO}_{8}$ : 527.1425; found: 527.1425 .

\section{3-Ethyl 2-(9H-Fluoren-9-ylmethyl) (3S)-6-Ethoxy-1,5,2-diox-} azinane-2,3-dicarboxylate (11)

IR (neat): 2674, 2986, 2901, 1741, 1450, 1355, 1176, $1057 \mathrm{~cm}^{-1}$.

${ }^{1} \mathrm{H}$ NMR $\left(500 \mathrm{MHz}, \mathrm{CDCl}_{3}\right): \delta=7.86-7.61(\mathrm{~m}, 4 \mathrm{H}), 7.47-7.31(\mathrm{~m}, 4 \mathrm{H})$, $5.43(\mathrm{~s}, 1 \mathrm{H}), 4.72-4.61(\mathrm{~m}, 2 \mathrm{H}), 4.57-4.31(\mathrm{~m}, 2 \mathrm{H}), 4.28(\mathrm{q}, J=7.1 \mathrm{~Hz}$, $2 \mathrm{H}$ ), 4.14-4.04 (m, $1 \mathrm{H}), 3.88$ (ddq, $J=44.5,9.6,7.1 \mathrm{~Hz}, 2 \mathrm{H}$ ), 1.37$1.24(\mathrm{~m}, 6 \mathrm{H}) ; 1 \mathrm{H}$ not observed.

${ }^{13} \mathrm{C}$ NMR (126 MHz, $\mathrm{CDCl}_{3}$ ): $\delta=167.46$ (CO), $156.01(\mathrm{CO}), 143.85(2$ C) $)^{*}, 143.31(2 \mathrm{C}), 141.50(2 \mathrm{C})^{*}, 141.44(2 \mathrm{C}), 128.02(2 \mathrm{CH})^{*}, 127.98(2$ $\mathrm{CH}), 127.26(2 \mathrm{CH})^{*}, 127.24(2 \mathrm{CH}), 125.50(2 \mathrm{CH})^{*}, 125.28(2 \mathrm{CH})$, $120.15(2 \mathrm{CH}), 113.06(\mathrm{CH}), 68.54\left(\mathrm{CH}_{2}\right), 64.90\left(\mathrm{CH}_{2}\right), 63.20\left(\mathrm{CH}_{2}\right)$, $62.45\left(\mathrm{CH}_{2}\right), 47.07(\mathrm{CH}), 15.28\left(\mathrm{CH}_{3}\right), 14.24\left(\mathrm{CH}_{3}\right)$; * signals of rotamers/diastereomers.; $1 \mathrm{C}$ not observed.

HRMS (ESI): $m / z\left[\mathrm{M}+\mathrm{NH}_{4}\right]^{+}$calcd for $\mathrm{C}_{23} \mathrm{H}_{29} \mathrm{~N}_{2} \mathrm{O}_{7}$ : 445.1969; found: 445.1966

\section{Fmoc Deprotection of Acid-Protected Monomer; General Proce- dure}

Piperidine (5.00 equiv) was added dropwise to Fmoc-protected monomer ( 1.00 equiv) in $\mathrm{CH}_{2} \mathrm{Cl}_{2}(0.025 \mathrm{M})$. The reaction was stirred for $1 \mathrm{~h}$ at r.t. The solution was diluted with $\mathrm{CH}_{2} \mathrm{Cl}_{2}$ and sat. aq $\mathrm{NH}_{4} \mathrm{Cl}$ solution was added. The phases were separated and the aqueous layer was extracted with $\mathrm{CH}_{2} \mathrm{Cl}_{2}(2 \times)$. The combined organic layers were 
washed with brine and dried $\left(\mathrm{Na}_{2} \mathrm{SO}_{4}\right)$. The drying agent was removed by filtration and the solvent was evaporated. The residue was purified by flash chromatography.

\section{Ethyl (3S)-6-(4-Bromophenyl)-1,5,2-dioxazinane-3-carboxylate (15)} IR (neat): 3674, 2986, 2901, 1732, 1490, 1369, 1210, $1043 \mathrm{~cm}^{-1}$.

${ }^{1} \mathrm{H} \mathrm{NMR}\left(500 \mathrm{MHz}, \mathrm{CDCl}_{3}\right): \delta=7.50-7.45(\mathrm{~m}, 2 \mathrm{H}), 7.32-7.28(\mathrm{~m}, 2 \mathrm{H})$, 6.86 (br s, $1 \mathrm{H}), 5.80(\mathrm{~s}, 1 \mathrm{H}), 4.66$ (dd, $J=11.4,1.4 \mathrm{~Hz}, 1 \mathrm{H}), 4.38-4.26$ (m, $3 \mathrm{H}), 3.52$ (dd, $J=3.2,1.4 \mathrm{~Hz}, 1 \mathrm{H}), 1.34(\mathrm{t}, J=7.1 \mathrm{~Hz}, 3 \mathrm{H})$.

${ }^{13} \mathrm{C} \mathrm{NMR}\left(126 \mathrm{MHz}, \mathrm{CDCl}_{3}\right): \delta=170.61(\mathrm{CO}), 135.50(\mathrm{C}), 131.53(2 \mathrm{CH})$, $128.30(2 \mathrm{CH}), 123.58(\mathrm{CBr}), 101.88(\mathrm{CH}), 65.27\left(\mathrm{CH}_{2}\right), 62.07\left(\mathrm{CH}_{2}\right)$, $58.68(\mathrm{CH}), 14.39\left(\mathrm{CH}_{3}\right)$.

HRMS (ESI): $m / z$ [M $+\mathrm{H}]^{+}$calcd for $\mathrm{C}_{12} \mathrm{H}_{15} \mathrm{BrNO}_{4}$ : 316.0179; found: 316.0178 .

Ethyl (3S)-6-(4-Nitrophenyl)-1,5,2-dioxazinane-3-carboxylate (16) IR (neat): 3674, 2987, 2901, 1734, 1522, 1348, 1214, $1044 \mathrm{~cm}^{-1}$.

${ }^{1} \mathrm{H} \mathrm{NMR}\left(500 \mathrm{MHz}, \mathrm{CDCl}_{3}\right): \delta=8.22-8.18(\mathrm{~m}, 2 \mathrm{H}), 7.64-7.56(\mathrm{~m}, 2 \mathrm{H})$, $6.94(\mathrm{~s}, 1 \mathrm{H}), 5.93(\mathrm{~s}, 1 \mathrm{H}), 4.70(\mathrm{dt}, J=11.4,1.4 \mathrm{~Hz}, 1 \mathrm{H}), 4.37-4.29(\mathrm{~m}$, $3 \mathrm{H}), 3.56$ (dd, $J=3.3,1.4 \mathrm{~Hz}, 1 \mathrm{H}), 1.34(\mathrm{t}, J=7.1 \mathrm{~Hz}, 3 \mathrm{H})$.

${ }^{13} \mathrm{C} \mathrm{NMR}\left(126 \mathrm{MHz}, \mathrm{CDCl}_{3}\right): \delta=170.45(\mathrm{CO}), 148.55\left(\mathrm{CNO}_{2}\right), 142.82$ (C), $127.64(2 \mathrm{CH}), 123.56(2 \mathrm{CH}), 100.98(\mathrm{CH}), 65.34\left(\mathrm{CH}_{2}\right), 62.20$ $\left(\mathrm{CH}_{2}\right), 58.73(\mathrm{CH}), 14.40\left(\mathrm{CH}_{3}\right)$.

HRMS (ESI): $m / z[\mathrm{M}+\mathrm{H}]^{+}$calcd for $\mathrm{C}_{12} \mathrm{H}_{15} \mathrm{~N}_{2} \mathrm{O}_{6}$ : 283.0925; found: 283.0927.

\section{Ethyl (3S)-6-Ethoxy-1,5,2-dioxazinane-3-carboxylate (17)}

IR (neat): 3674, 2987, 2900, 1736, 1393, 1250, 1074, $1056 \mathrm{~cm}^{-1}$.

${ }^{1} \mathrm{H}$ NMR $\left(500 \mathrm{MHz}, \mathrm{CDCl}_{3}\right): \delta=6.37(\mathrm{~d}, J=119.9 \mathrm{~Hz}, 1 \mathrm{H}), 5.43(\mathrm{dd}, J=$ $11.2,0.5 \mathrm{~Hz}, 1 \mathrm{H}), 4.43-4.35(\mathrm{~m}, 1 \mathrm{H}), 4.26$ (p, J = 7.2 Hz, $2 \mathrm{H}), 4.09-$ $4.01(\mathrm{~m}, 1 \mathrm{H}), 3.89-3.66(\mathrm{~m}, 3 \mathrm{H}), 1.33-1.28$ (m, $3 \mathrm{H}), 1.28-1.23$ (m, 3 $\mathrm{H})$.

${ }^{13} \mathrm{C}$ NMR (126 MHz, $\mathrm{CDCl}_{3}$ ): $\delta=169.44(\mathrm{CO})^{*}, 168.41(\mathrm{CO}), 111.02$ $(\mathrm{CH})^{*}, 110.47(\mathrm{CH}), 62.92\left(\mathrm{CH}_{2}\right)^{*}, 62.69\left(\mathrm{CH}_{2}\right), 62.63\left(\mathrm{CH}_{2}\right)^{*}, 62.46$ $\left(\mathrm{CH}_{2}\right), 61.97\left(\mathrm{CH}_{2}\right)^{*}, 61.89\left(\mathrm{CH}_{2}\right), 57.65(\mathrm{CH})^{*}, 57.53(\mathrm{CH}), 15.16\left(\mathrm{CH}_{3}\right)$, $14.28\left(\mathrm{CH}_{3}\right)^{*}, 14.26\left(\mathrm{CH}_{3}\right)$; * signals of diastereomers.

HRMS (ESI): $m / z$ [M + Na] ${ }^{+}$calcd for $\mathrm{C}_{8} \mathrm{H}_{15} \mathrm{NNaO}_{5}: 228.0842$; found: 228.0842 .

\section{Ethyl (S)-5,9-Dioxa-6-azaspiro[3.5]nonane-7-carboxylate (18)}

IR (neat): 3674, 2987, 2901, 1733, 1451, 1375, 1280, 1079, $1020 \mathrm{~cm}^{-1}$. ${ }^{1} \mathrm{H}$ NMR $\left(500 \mathrm{MHz}, \mathrm{CDCl}_{3}\right): \delta=4.26(\mathrm{q}, J=7.2 \mathrm{~Hz}, 2 \mathrm{H}), 4.07(\mathrm{~d}, J=4.4$ $\mathrm{Hz}, 2 \mathrm{H}), 3.67$ (t, $J=4.4 \mathrm{~Hz}, 1 \mathrm{H}), 2.44-2.14(\mathrm{~m}, 4 \mathrm{H}), 1.81-1.71(\mathrm{~m}, 2$ $\mathrm{H}), 1.30(\mathrm{t}, J=7.1 \mathrm{~Hz}, 3 \mathrm{H})$; NH not observed.

${ }^{13} \mathrm{C}$ NMR $\left(126 \mathrm{MHz}, \mathrm{CDCl}_{3}\right): \delta=169.68(\mathrm{CO}), 103.05(\mathrm{C}), 61.79\left(\mathrm{CH}_{2}\right)$, $60.59\left(\mathrm{CH}_{2}\right), 58.37(\mathrm{CH}), 32.30\left(\mathrm{CH}_{2}\right), 31.64\left(\mathrm{CH}_{2}\right), 14.28\left(\mathrm{CH}_{3}\right), 11.85$ $\left(\mathrm{CH}_{2}\right)$.

HRMS (ESI): $m / z[M+H]^{+}$calcd for $\mathrm{C}_{9} \mathrm{H}_{16} \mathrm{NO}_{4}$ : 202.1074; found: 202.1074.

7-Benzyl 2-Methyl (S)-5,9-Dioxa-6-azaspiro[3.5]nonane-2,7-dicarboxylate (24)

IR (neat): 3674, 2987, 2900, 2253, 1733, 1453, 1393, 1258, 1065, 903 $\mathrm{cm}^{-1}$.
${ }^{1} \mathrm{H}$ NMR $\left(500 \mathrm{MHz}, \mathrm{CDCl}_{3}\right): \delta=7.45-7.27(\mathrm{~m}, 5 \mathrm{H}), 6.18(\mathrm{~s}, 1 \mathrm{H}), 5.29-$ $5.21(\mathrm{~m}, 2 \mathrm{H}), 4.21-4.03(\mathrm{~m}, 2 \mathrm{H}), 3.70(\mathrm{~d}, J=1.1 \mathrm{~Hz}, 3 \mathrm{H}), 3.66(\mathrm{t}, J=$ $3.8 \mathrm{~Hz}, 1 \mathrm{H}), 2.99-2.40(\mathrm{~m}, 5 \mathrm{H})$.

${ }^{13} \mathrm{C}$ NMR (126 MHz, $\mathrm{CDCl}_{3}$ ): $\delta=175.15$ (CO), 169.67 (CO), 135.29 (C), $128.78(2 \mathrm{CH}), 128.66(\mathrm{CH}), 128.39(2 \mathrm{CH})^{*}, 128.37(2 \mathrm{CH}), 100.02$ $(\mathrm{C})^{*}, 99.91(\mathrm{C}), 67.47\left(\mathrm{CH}_{2}\right), 60.68\left(\mathrm{CH}_{2}\right)^{*}, 60.61\left(\mathrm{CH}_{2}\right), 58.49(\mathrm{CH})^{*}$, $58.41(\mathrm{CH}), 52.14(\mathrm{CH})^{*}, 52.12(\mathrm{CH}), 36.54\left(\mathrm{CH}_{2}\right)^{*}, 36.37\left(\mathrm{CH}_{2}\right), 35.54$ $\left(\mathrm{CH}_{2}\right)^{*}, 35.31\left(\mathrm{CH}_{2}\right), 29.09(\mathrm{CH})^{*}, 28.96(\mathrm{CH})$; ${ }^{*}$ signals of rotamers/diastereomers.

HRMS (ESI): $m / z[M+H]^{+}$calcd for $\mathrm{C}_{16} \mathrm{H}_{20} \mathrm{NO}_{6}$ : 322.1285; found: 322.1286

\section{Benzyl (S)-2-Bromo-5,9-dioxa-6-azaspiro[3.5]nonane-7-carboxyl- ate (25)}

IR (neat): 3674, 3296, 2987, 2900, 1738, 1454, 1282, 1198, $1065 \mathrm{~cm}^{-1}$. ${ }^{1} \mathrm{H}$ NMR $\left(500 \mathrm{MHz}, \mathrm{CDCl}_{3}\right): \delta=7.44-7.33(\mathrm{~m}, 5 \mathrm{H}), 6.23(\mathrm{~s}, 1 \mathrm{H}), 5.33-$ $5.23(\mathrm{~m}, 2 \mathrm{H}), 4.33-4.13(\mathrm{~m}, 2 \mathrm{H}), 4.10(\mathrm{ddd}, J=11.5,3.4,2.7 \mathrm{~Hz}, 1 \mathrm{H})$, $3.68(\mathrm{dt}, J=7.7,3.9 \mathrm{~Hz}, 1 \mathrm{H}), 3.37-2.57(\mathrm{~m}, 4 \mathrm{H})$.

${ }^{13} \mathrm{C} \mathrm{NMR}\left(126 \mathrm{MHz}, \mathrm{CDCl}_{3}\right.$ ): $\delta=169.55(\mathrm{CO})^{*}, 169.45(\mathrm{CO}), 135.23(\mathrm{C})$, $128.80(2 \mathrm{CH}), 128.71(\mathrm{CH}), 128.41(2 \mathrm{CH})^{*}, 128.39(2 \mathrm{CH}), 100.62(\mathrm{C})$, $67.55\left(\mathrm{CH}_{2}\right), 60.88\left(\mathrm{CH}_{2}\right), 58.44(\mathrm{CH})^{*}, 58.41(\mathrm{CH}), 45.95\left(\mathrm{CH}_{2}\right)^{*}, 45.78$ $\left(\mathrm{CH}_{2}\right), 44.90\left(\mathrm{CH}_{2}\right)^{*}, 44.57\left(\mathrm{CH}_{2}\right), 32.98(\mathrm{CH})^{*}, 32.75(\mathrm{CH}){ }^{*}$ signals of rotamers/diastereomers.

HRMS (ESI): $m / z$ [M $+\mathrm{H}]^{+}$calcd for $\mathrm{C}_{14} \mathrm{H}_{17} \mathrm{BrNO}_{4}$ : 342.0335; found: 342.0331 .

\section{Benzyl Deprotection; General Procedure}

$\mathrm{Pd}(\mathrm{OH})_{2} /$ charcoal $(20 \%$ on carbon wetted with ca. $50 \%$ water) $(6 \%$ $\mathrm{m} / \mathrm{m}$ ) was added to a solution of benzyl-protected acid (1.00 equiv) in $\mathrm{CH}_{2} \mathrm{Cl}_{2} / \mathrm{EtOH}(1: 4)$ at $0{ }^{\circ} \mathrm{C}$. The mixture was stirred under $\mathrm{H}_{2}$ atmosphere for $1 \mathrm{~h}$ while gradually warming up to r.t. The mixture was filtered through a plug of celite and the solvent was evaporated.

\section{(S)-6-[(9H-Fluoren-9-ylmethoxy)carbonyl]-5,9-dioxa-6- azaspiro[3.5]nonane-7-carboxylic Acid (26)}

IR (neat): 3674, 2987, 2900, 1717, 1450, 1394, 1266, 1133, $1065 \mathrm{~cm}^{-1}$. ${ }^{1} \mathrm{H}$ NMR (500 MHz, $\left.\mathrm{CDCl}_{3}\right): \delta=8.65$ (br s, $\left.1 \mathrm{H}\right), 7.79-7.73(\mathrm{~m}, 2 \mathrm{H})$, $7.64(\mathrm{~d}, J=45.5 \mathrm{~Hz}, 2 \mathrm{H}), 7.44-7.37(\mathrm{~m}, 2 \mathrm{H}), 7.31(\mathrm{tt}, J=7.4,1.1 \mathrm{~Hz}, 2$ $\mathrm{H}), 4.66(\mathrm{dd}, J=10.5,6.2 \mathrm{~Hz}, 1 \mathrm{H}), 4.49(\mathrm{~s}, 2 \mathrm{H}), 4.38-4.22(\mathrm{~m}, 2 \mathrm{H})$, $4.01(\mathrm{~s}, 1 \mathrm{H}), 2.44-2.18(\mathrm{~m}, 4 \mathrm{H}), 1.87$ (dtd, $J=19.3,12.1,10.7,7.0 \mathrm{~Hz}$, $2 \mathrm{H})$.

${ }^{13} \mathrm{C}$ NMR (126 MHz, $\mathrm{CDCl}_{3}$ ): $\delta=173.61$ (CO), $156.39(\mathrm{CO}), 143.82(2$ C) $)^{*}, 143.19$ (2 C), $141.50(2 \mathrm{C})^{*}, 141.48(2 \mathrm{C}), 128.05(2 \mathrm{CH})^{*}, 127.98(2$ $\mathrm{CH}), 127.39(2 \mathrm{CH})^{*}, 127.17(2 \mathrm{CH}), 125.42(2 \mathrm{CH})^{*}, 125.08(2 \mathrm{CH})$, $120.14(2 \mathrm{CH}), 105.24(\mathrm{C}), 68.62\left(\mathrm{CH}_{2}\right), 60.08\left(\mathrm{CH}_{2}\right), 56.89(\mathrm{CH}), 46.99$ (CH), $32.60\left(\mathrm{CH}_{2}\right), 30.25\left(\mathrm{CH}_{2}\right), 11.47\left(\mathrm{CH}_{2}\right)$; * signals of rotamers.

HRMS (ESI): $m / z[\mathrm{M}+\mathrm{Na}]^{+}$calcd for $\mathrm{C}_{22} \mathrm{H}_{21} \mathrm{NNaO}_{6}: 418.1261$; found: 418.1261.

(S)-6-[(9H-Fluoren-9-ylmethoxy)carbonyl]-2-(methoxycarbonyl)5,9-dioxa-6-azaspiro[3.5]nonane-7-carboxylic Acid (27)

IR (neat): 3674, 2987, 2900, 1730, 1606, 1450, 1266, 1224, $1065 \mathrm{~cm}^{-1}$. ${ }^{1} \mathrm{H}$ NMR $\left(400 \mathrm{MHz}, \mathrm{CDCl}_{3}\right): \delta=7.78-7.73(\mathrm{~m}, 2 \mathrm{H}), 7.72-7.49(\mathrm{~m}, 2 \mathrm{H})$, 7.45-7.28 (m, $4 \mathrm{H}), 4.70-4.25(\mathrm{~m}, 5 \mathrm{H}), 4.14-3.91(\mathrm{~m}, 1 \mathrm{H}), 3.71(\mathrm{~d}, J=$ $20.1 \mathrm{~Hz}, 3 \mathrm{H}), 3.10-2.25(\mathrm{~m}, 4 \mathrm{H})$.

${ }^{13} \mathrm{C}$ NMR (101 MHz, $\left.\mathrm{CDCl}_{3}\right): \delta=174.71(\mathrm{CO})^{*}, 174.37(\mathrm{CO}), 172.75$ (CO)*, 172.68 (CO), 158.09 (CO), $143.85(2 \mathrm{C})^{*}, 143.33(2 \mathrm{C})^{*}, 143.19(2$ C), $141.50(2 \mathrm{C})^{*}, 141.49(2 \mathrm{C})^{*}, 141.43(2 \mathrm{C})^{*}, 141.40$ (2 C), 128.04 (2 
$\mathrm{CH})^{*}, 127.99(2 \mathrm{CH}), 127.95(\mathrm{CH}), 127.39(2 \mathrm{CH})^{*}, 127.37(2 \mathrm{CH})$, $127.19(2 \mathrm{CH})^{*}, 127.06(2 \mathrm{CH})^{*}, 127.04(2 \mathrm{CH}), 125.61(2 \mathrm{CH})^{*}, 125.32$ $(2 \mathrm{CH})^{*}, 125.25(2 \mathrm{CH})^{*}, 125.02(2 \mathrm{CH}), 120.16(2 \mathrm{CH})^{*}, 120.09(2 \mathrm{CH})$, $101.88(\mathrm{C})^{*}, 101.65(\mathrm{C}), 68.97\left(\mathrm{CH}_{2}\right)^{*}, 68.34\left(\mathrm{CH}_{2}\right), 60.52\left(\mathrm{CH}_{2}\right)^{*}, 60.42$ $\left(\mathrm{CH}_{2}\right), 52.30\left(\mathrm{CH}_{3}\right), 47.06(\mathrm{CH})^{*}, 46.89(\mathrm{CH}), 36.54\left(\mathrm{CH}_{2}\right)^{*}, 36.37\left(\mathrm{CH}_{2}\right)$, $34.60\left(\mathrm{CH}_{2}\right)^{*}, 34.20\left(\mathrm{CH}_{2}\right), 28.79(\mathrm{CH}), 28.67(\mathrm{C})$; * signals of rotamers/diastereomers.

HRMS (MALDI/ESI): $m / z$ [M + Na $]^{+}$calcd for $\mathrm{C}_{24} \mathrm{H}_{23} \mathrm{NNaO}_{8}$ : 476.1316; found: 476.1316 .

(S)-2-Bromo-6-[(9H-fluoren-9-ylmethoxy)carbonyl]-5,9-dioxa-6azaspiro[3.5]nonane-7-carboxylic Acid (28)

IR (neat): 3674, 2987, 2900, 1717, 1450, 1408, 1283, 1264, 1139, 1067 $\mathrm{cm}^{-1}$.

${ }^{1} \mathrm{H}$ NMR $\left(400 \mathrm{MHz}, \mathrm{CDCl}_{3}\right): \delta=9.23(\mathrm{br} \mathrm{s}, 1 \mathrm{H}), 7.82-7.73(\mathrm{~m}, 2 \mathrm{H})$, 7.59 (dd, $J=24.6,7.5 \mathrm{~Hz}, 2 \mathrm{H}), 7.46-7.28(\mathrm{~m}, 4 \mathrm{H}), 4.67(\mathrm{~d}, J=5.9 \mathrm{~Hz}, 2$ H), 4.36-4.21 (m, $2 \mathrm{H}), 4.20-3.97(\mathrm{~m}, 1 \mathrm{H}), 3.96-3.85(\mathrm{~m}, 1 \mathrm{H}), 2.95(\mathrm{~d}$, $J=15.7 \mathrm{~Hz}, 2 \mathrm{H}), 2.70-2.45(\mathrm{~m}, 2 \mathrm{H}) ; 1 \mathrm{H}$ not observed.

${ }^{13} \mathrm{C}$ NMR (101 MHz, $\mathrm{CDCl}_{3}$ ): $\delta=173.11(\mathrm{CO}), 155.77$ (CO), $143.71(2$ C)*, $143.08(2 \mathrm{C}), 141.55(2 \mathrm{C})^{*}, 141.51(2 \mathrm{C}), 128.09(2 \mathrm{CH})^{*}, 128.05(2$ $\mathrm{CH}), 127.37(2 \mathrm{CH})^{*}, 127.26(2 \mathrm{CH}), 125.05(2 \mathrm{CH})^{*}, 124.90(2 \mathrm{CH})$, $120.21(2 \mathrm{CH})^{*}, 120.15(2 \mathrm{CH}), 102.49(\mathrm{C}), 68.01\left(\mathrm{CH}_{2}\right), 60.71\left(\mathrm{CH}_{2}\right)$, $47.16(\mathrm{CH}), 45.42\left(\mathrm{CH}_{2}\right), 43.85\left(\mathrm{CH}_{2}\right), 31.46(\mathrm{CH})$; * signals of rotamers/diastereomers; $1 \mathrm{C}$ not observed.

HRMS (MALDI/ESI): $m / z[\mathrm{M}+\mathrm{Na}]^{+}$calcd for $\mathrm{C}_{22} \mathrm{H}_{20} \mathrm{BrNNaO}_{6}$ : 496.0366; found: 496.0367 .

(S)-2-(tert-Butoxycarbonyl)-6-[(9H-fluoren-9-ylmethoxy)carbonyl]-5,9-dioxa-6-azaspiro[3.5]nonane-7-carboxylic Acid (29)

IR (neat): 3674, 2976, 2901, 1719, 1450, 1366, 1283, 1135, $1060 \mathrm{~cm}^{-1}$. ${ }^{1} \mathrm{H} \mathrm{NMR}\left(400 \mathrm{MHz}, \mathrm{CDCl}_{3}\right): \delta=7.80-7.55(\mathrm{~m}, 4 \mathrm{H}), 7.44-7.28(\mathrm{~m}, 4 \mathrm{H}$ ), 4.74-3.61 (m, $6 \mathrm{H}$ ), 3.03-2.01 (m, $5 \mathrm{H}$ ), 1.46 (d, J = $19.5 \mathrm{~Hz}, 9 \mathrm{H}$ ); OH not observed.

${ }^{13} \mathrm{C}$ NMR (101 MHz, $\mathrm{CDCl}_{3}$ ): $\delta=173.54(\mathrm{CO}) * 173.23(\mathrm{CO}), 173.10$ $(\mathrm{CO})^{*}, 173.01(\mathrm{CO}), 156.42(\mathrm{CO}), 143.86(2 \mathrm{C})^{*}, 143.32(2 \mathrm{C})^{*}, 143.19(2$ C), $141.49(2 \mathrm{C})^{*}, 141.42(2 \mathrm{C})^{*}, 141.37(2 \mathrm{C}), 128.03(2 \mathrm{CH})^{*}, 127.96(2$ $\mathrm{CH})^{*}, 127.92(2 \mathrm{CH}), 127.41(2 \mathrm{CH})^{*}, 127.37(2 \mathrm{CH})^{*}, 127.19(2 \mathrm{CH})$, $125.66(2 \mathrm{CH})^{*}, 125.34(2 \mathrm{CH})^{*}, 125.04(2 \mathrm{CH}), 120.16(2 \mathrm{CH})^{*}, 120.05$ $(2 \mathrm{CH}), 101.97(\mathrm{C}), 101.65(\mathrm{C}), 81.06(\mathrm{C})^{*}, 81.01(\mathrm{C}), 68.91\left(\mathrm{CH}_{2}\right)^{*}$, $68.34\left(\mathrm{CH}_{2}\right), 60.44\left(\mathrm{CH}_{2}\right)^{*}, 60.38\left(\mathrm{CH}_{2}\right), 57.20(\mathrm{CH}), 47.04(\mathrm{CH}), 46.88$ $(\mathrm{CH}), 36.49\left(\mathrm{CH}_{2}\right)^{*}, 36.21\left(\mathrm{CH}_{2}\right), 34.49\left(\mathrm{CH}_{2}\right)^{*}, 34.01\left(\mathrm{CH}_{2}\right), 29.93(\mathrm{CH})^{*}$, $29.70(\mathrm{CH}), 28.18\left(3 \mathrm{CH}_{3}\right)$; ${ }^{*}$ signals of rotamers/diastereomers.

HRMS (MALDI/ESI): $m / z[\mathrm{M}+\mathrm{Na}]^{+}$calcd for $\mathrm{C}_{27} \mathrm{H}_{29} \mathrm{NNaO}_{8}: 518.1785$; found: 518.1783 .

\section{KAHA Ligation; General Procedure}

$\alpha$-Ketoacid 13 ( 1.20 equiv) was added to the free hydroxylamine $(1.00$ equiv) and dissolved in $\mathrm{HFIP} / \mathrm{AcOH}(1: 1,30 \mathrm{mM})$ containing $1 \%$ water. The mixture was heated to $45^{\circ} \mathrm{C}$ for $12 \mathrm{~h}$ with monitoring by analytical HPLC. The crude mixture was directly purified by preparative HPLC (Shiseido Capcell Pak C18 column, $50 \times 250 \mathrm{~mm}$ ) heated to $60{ }^{\circ} \mathrm{C}$ using a gradient of 5 to $80 \% \mathrm{MeCN}$ in Milipore- $\mathrm{H}_{2} \mathrm{O}$ containing $0.1 \%$ $(\mathrm{v} / \mathrm{v})$ TFA over $35 \mathrm{~min}$. HPLC monitoring of the KAHA ligation was carried out using Shiseido Capcell Pak UG80 C18 column $(4.6 \times 250 \mathrm{~mm})$, heated to $60{ }^{\circ} \mathrm{C}$, using a gradient of 10 to $90 \% \mathrm{MeCN}$ in Milipore- $\mathrm{H}_{2} \mathrm{O}$ containing $0.1 \%(\mathrm{v} / \mathrm{v})$ TFA over $20 \mathrm{~min}$.
Ethyl $\mathbf{N}$-[3-(4-Nitrophenyl)propanoyl]-L-serinate (14)

IR (neat): 3384, 2987, 2901, 1734, 1646, 1517, 1451, 1346, 1228, 1056 $\mathrm{cm}^{-1}$.

${ }^{1} \mathrm{H}$ NMR (500 MHz, MeOD): $\delta=8.19-8.13(\mathrm{~m}, 2 \mathrm{H}), 7.51-7.46(\mathrm{~m}, 2 \mathrm{H})$, $4.48(\mathrm{dd}, J=5.1,4.2 \mathrm{~Hz}, 1 \mathrm{H}), 4.18(\mathrm{qd}, J=7.2,2.2 \mathrm{~Hz}, 2 \mathrm{H}), 3.88-3.71$ (m, $2 \mathrm{H}), 3.07$ (td, $J=7.4,1.3 \mathrm{~Hz}, 2 \mathrm{H}), 2.65(\mathrm{td}, J=7.5,2.1 \mathrm{~Hz}, 2 \mathrm{H})$, $1.25(\mathrm{t}, J=7.1 \mathrm{~Hz}, 3 \mathrm{H})$.

${ }^{13} \mathrm{C}$ NMR (126 MHz, MeOD): $\delta=174.56$ (CO), $171.76(\mathrm{CO}), 150.32(\mathrm{C})$, 147.97 $\left(\mathrm{CNO}_{2}\right), 130.61(2 \mathrm{CH}), 124.53(2 \mathrm{CH}), 62.80\left(\mathrm{CH}_{2}\right), 62.46\left(\mathrm{CH}_{2}\right)$, $56.27(\mathrm{CH}), 37.50\left(\mathrm{CH}_{2}\right), 32.27\left(\mathrm{CH}_{2}\right), 14.43\left(\mathrm{CH}_{3}\right)$.

HRMS (ESI): $m / z$ [M + Na] ${ }^{+}$calcd for $\mathrm{C}_{14} \mathrm{H}_{18} \mathrm{~N}_{2} \mathrm{NaO}_{6}: 333.1057$; found: 333.1061.

Benzyl N-(3-(4-Nitrophenyl)propanoyl)-L-serinate (23)

IR (neat): 2939, 1737, 1643, 1515, 1454, 1343, $1190 \mathrm{~cm}^{-1}$.

${ }^{1} \mathrm{H}$ NMR $(500 \mathrm{MHz}, \mathrm{MeOD}): \delta=8.14-8.10(\mathrm{~m}, 2 \mathrm{H}), 7.47-7.43(\mathrm{~m}, 2 \mathrm{H})$, 7.38-7.28 (m, $5 \mathrm{H}), 5.22-5.13(\mathrm{~m}, 2 \mathrm{H}), 4.55$ (dd, J = 5.2, $4.2 \mathrm{~Hz}, 1 \mathrm{H})$, 3.90-3.73 (m, $2 \mathrm{H}), 3.09-2.99(\mathrm{~m}, 2 \mathrm{H}), 2.63$ (td, $J=7.5,2.3 \mathrm{~Hz}, 2 \mathrm{H})$.

${ }^{13} \mathrm{C}$ NMR (126 MHz, MeOD): $\delta=174.64$ (CO), $171.64(\mathrm{CO}), 150.28(\mathrm{C})$, $147.95\left(\mathrm{CNO}_{2}\right), 137.20(\mathrm{C}), 130.57(2 \mathrm{CH}), 129.54(2 \mathrm{CH}), 129.27(\mathrm{CH})$, $129.11(2 \mathrm{CH}), 124.52(2 \mathrm{CH}), 68.00\left(\mathrm{CH}_{2}\right), 62.75\left(\mathrm{CH}_{2}\right), 56.35(\mathrm{CH})$, $37.47\left(\mathrm{CH}_{2}\right), 32.26\left(\mathrm{CH}_{2}\right)$.

HRMS (ESI): $m / z[\mathrm{M}+\mathrm{Na}]^{+}$calcd for $\mathrm{C}_{19} \mathrm{H}_{20} \mathrm{~N}_{2} \mathrm{NaO}_{6}$ : 395.1214.1057; found: 395.1218 .

(S)-N-(2-amino-2-oxoethyl)-2-(2-((2S,5S)-6-hydroxy-5-(3-(4-nitrophenyl)propanamido)-3,4-dioxohexan-2-yl)hydrazineyl)-3-(4hydroxyphenyl)propanamide (32)

HRMS (ESI): $m / z$ [M + Na] ${ }^{+}$calcd for $\mathrm{C}_{26} \mathrm{H}_{32} \mathrm{~N}_{6} \mathrm{NaO}_{9}$ : 595.2123; found: 595.2123.

HPLC (Shiseido Capcell Pak UG80 C18 column $(4.6 \times 250 \mathrm{~mm})$, heated to $60{ }^{\circ} \mathrm{C}, 10$ to $90 \% \mathrm{CH}_{3} \mathrm{CN}$ with $0.1 \% \mathrm{TFA}$ in $20 \mathrm{~min}$ ): $t_{\mathrm{R}}=19.8 \mathrm{~min}$.

\section{Segment 36}

HRMS (ESI): $m / z$ [M] $]^{+}$calcd for $\mathrm{C}_{263} \mathrm{H}_{418} \mathrm{~N}_{70} \mathrm{O}_{81} \mathrm{~S}_{2}$ : 5917.0183; found: 5918.0125.

HPLC (Shiseido Capcell Pak UG80 C18 column ( $4.6 \times 250 \mathrm{~mm}$ ), heated to $60{ }^{\circ} \mathrm{C}, 10$ to $90 \% \mathrm{CH}_{3} \mathrm{CN}$ with $0.1 \% \mathrm{TFA}$ in $20 \mathrm{~min}$ ): $t_{\mathrm{R}}=22.4 \mathrm{~min}$.

\section{Funding Information}

This work was supported by the Swiss National Science Foundation (169451).

\section{Acknowledgment}

We thank the MS and NMR services of the Laboratorium für Organische Chemie at ETH Zürich for analyses and Haewon Song for correcting the manuscript.

\section{Supporting Information}

Supporting information for this article is available online at https://doi.org/10.1055/s-0037-1611635. 


\section{References}

(1) Bode, J. W.; Fox, R. M.; Baucom, K. D. Angew. Chem. Int. Ed. 2006, $45,1248$.

(2) Dawson, P. E.; Muir, T. W.; Clark-Lewis, I.; Kent, S. B. Science (Washington, D. C.) 1994, 266, 776.

(3) Other amide-forming ligations include the traceless Staudinger and the serine/threonine ligation: (a) Kleineweischede, R.; Hackenberger, C. P. R. Angew. Chem. Int. Ed. 2008, 47, 5984. (b) Zhang, Y.; Xu, C.; Lam, H. Y.; Lee, C. L.; Li, X. Proc. Natl. Acad. Sci. U.S.A. 2013, 110, 6657.

(4) Pattabiraman, V. R.; Ogunkoya, A. O.; Bode, J. W. Angew. Chem. Int. Ed. 2012, 51, 5114.

(5) Wucherpfennig, T. G.; Rohrbacher, F.; Pattabiraman, V. R.; Bode, J. W. Angew. Chem. Int. Ed. 2014, 53, 12244.

(6) Pusterla, I.; Bode, J. W. Nat. Chem. 2015, 7, 668.
(7) (a) Tokuyama, H.; Kuboyama, T.; Amano, A.; Yamashita, T.; Fukuyama, T. Synthesis 2000, 1299. (b) Tokuyama, H.; Kuboyama, T.; Fukuyama, T. Org. Synth. 2003, 80, 207. (c) Medina, S. I.; Wu, J.; Bode, J. W. Org. Biomol. Chem. 2010, 8 , 3405.

(8) (a) Knowles, D. A.; Mathews, C. J.; Tomkinson, N. C. O. Synlett 2008, 2769. (b) Knight, D. W.; Leese, M. P. Tetrahedron Lett. 2001, 42, 2593. (c) Jones, K. L.; Porzelle, A.; Hall, A.; Woodrow, M. D.; Tomkinson, N. C. O. Org. Lett. 2008, 10, 797. (d) Blakemore, P. R. Science of Synthesis; Georg Thieme Verlag: Stuttgart, 2005, 857.

(9) Rohrbacher, F.; Baldauf, S.; Wucherpfennig, T. G.; Bode, J. W. Synlett 2017, 28, 1929.

(10) (a) Greene, T. W.; Wuts, P. G. M. Protective Groups in Organic Synthesis; John Wiley \& Sons: New York, 1999, 4rd ed. 300-302. (b) Rich, R. H.; Bartlett, P. A. J. Org. Chem. 1996, 61, 3916.

(11) Rohrbacher, F.; Zwicky, A.; Bode, J. W. Chem. Sci. 2017, 8, 4051. 